\title{
CNS-directed gene therapy for the treatment of neurologic and somatic mucopolysaccharidosis type II (Hunter syndrome)
}

\author{
Sandra Motas, ${ }^{1,2}$ Virginia Haurigot, $, 1,2,3$ Miguel Garcia, ${ }^{1,2,3}$ Sara Marcó, ${ }^{1,2}$ Albert Ribera, ${ }^{1,2,3}$ \\ Carles Roca, ${ }^{1,2,3}$ Xavier Sánchez, ${ }^{1,2}$ Víctor Sánchez, ${ }^{1,2}$ Maria Molas, ${ }^{1,2,3}$ Joan Bertolin, ${ }^{1,2}$ \\ Luca Maggioni, ${ }^{1,2,3}$ Xavier León, ${ }^{1,2,3}$ Jesús Ruberte, ${ }^{1,3,4}$ and Fatima Bosch ${ }^{1,2,3}$ \\ ${ }^{1}$ Center of Animal Biotechnology and Gene Therapy and ${ }^{2}$ Department of Biochemistry and Molecular Biology, \\ Universitat Autònoma de Barcelona, Bellaterra, Spain. ${ }^{3}$ Centro de Investigación Biomédica en Red de Diabetes y \\ Enfermedades Metabólicas Asociadas, Madrid, Spain. ${ }^{4}$ Department of Animal Health and Anatomy, School of Veterinary \\ Medicine, Universitat Autònoma de Barcelona, Bellaterra, Spain.
}

\begin{abstract}
Mucopolysaccharidosis type II (MPSII) is an X-linked lysosomal storage disease characterized by severe neurologic and somatic disease caused by deficiency of iduronate-2-sulfatase (IDS), an enzyme that catabolizes the glycosaminoglycans heparan and dermatan sulphate. Intravenous enzyme replacement therapy (ERT) currently constitutes the only approved therapeutic option for MPSII. However, the inability of recombinant IDS to efficiently cross the blood-brain barrier (BBB) limits ERT efficacy in treating neurological symptoms. Here, we report a gene therapy approach for MPSII through direct delivery of vectors to the CNS. Through a minimally invasive procedure, we administered adeno-associated virus vectors encoding IDS (AAV9-Ids) to the cerebrospinal fluid of MPSII mice with already established disease. Treated mice showed a significant increase in IDS activity throughout the encephalon, with full resolution of lysosomal storage lesions, reversal of lysosomal dysfunction, normalization of brain transcriptomic signature, and disappearance of neuroinflammation. Moreover, our vector also transduced the liver, providing a peripheral source of therapeutic protein that corrected storage pathology in visceral organs, with evidence of cross-correction of nontransduced organs by circulating enzyme. Importantly, AAV9-Ids-treated MPSII mice showed normalization of behavioral deficits and considerably prolonged survival. These results provide a strong proof of concept for the clinical translation of our approach for the treatment of Hunter syndrome patients with cognitive impairment.
\end{abstract}

Authorship note: S. Motas and V. Haurigot contributed equally to this work.

Conflict of interest: S. Motas, V. Haurigot, and F. Bosch are coinventors on a patent application (EP15382297) for the use of AAV vectors for the treatment of MPSII.

Submitted: January 25, 2016 Accepted: May 12, 2016 Published: June 9, 2016

Reference information: JCI Insight. 2016;1(9):e86696. doi:10.1172/jci.insight.86696.

\section{Introduction}

Mucopolysaccharidosis type II (MPSII), or Hunter syndrome, is an X-linked recessive lysosomal storage disease (LSD) caused by deficiency of iduronate-2-sulfatase (IDS) $(1,2)$. This enzyme is essential for the degradation of the glycosaminoglycans (GAGs), heparan and dermatan sulphate, and when nonfunctional or absent, the degradation of these compounds is compromised, leading to pathological accumulation (1). Hunter syndrome has a worldwide incidence of $0.30-0.71$ cases/100,000 live births (3), and it is the most prevalent MPS disorder in Asia $(4,5)$.

MPSII is a variable, progressive, and multisystemic disorder. Within a wide spectrum of clinical manifestations, MPSII is generally categorized in two main clinical forms based on age at onset, presence or absence of neurological involvement, and survival $(1,2)$. The most severe form is the most frequent; it has an onset of between 18 months and 4 years of age $(2,6,7)$ and is characterized by significant neurological involvement, manifested primarily as progressive cognitive deterioration (8). Severely affected children also show coarse facial features, skeletal deformities that affect mobility, recurrent respiratory infections, cardiac failure, and hepatosplenomegaly and die from a combination of neurological deterioration and cardiorespiratory failure in the mid-teenage years $(1,2)$.

Until recently, there were no specific approved therapies for MPSII. Therapeutic options rely on the 
principle of cross-correction, by which cells can uptake mannose-6-phosphate-tagged (M6P-tagged) soluble lysosomal enzymes from the extracellular compartment via M6P receptors located on the plasma membrane and target them to their lysosomes (9). Hematopoietic stem cell transplantation (HSCT) has proved efficient in the treatment of other forms of MPS (10-12). The degree of success in MPSII patients has, however, been very variable (13-16). The high morbidity and mortality associated with HSCT and the lack of clear evidence supporting neurocognitive benefits have limited its use in Hunter syndrome patients $(17,18)$. Since FDA approval, enzyme replacement therapy (ERT) with idursulfase (ELAPRASE, Shire Pharmaceuticals) has been indicated for treatment of MPSII. Some improvement in indicators of somatic disease has been demonstrated by weekly i.v. infusions of ELAPRASE (19, 20). Major drawbacks of the therapy are (a) potential development of serious anaphylactic reactions up to 24 hours after infusion (19, 20); (b) high incidence of development of anti-IDS antibodies (up to $50 \%$ of treated patients), which might limit long-term product efficacy (19-21); and (c) the inability of recombinant IDS to cross the brood-brain barrier (BBB) and reach the CNS following i.v. delivery, limiting the potential applicability to treat neurological symptoms. A recent study has evaluated the delivery of IDS to the cerebrospinal fluid (CSF) of cognitively impaired Hunter syndrome patients via an indwelling intrathecal drug delivery device (IDDD) (NCT00920647, https://clinicaltrials.gov/). Reductions of $80 \%-90 \%$ in CSF GAG levels were reported after 6 months of treatment (22). However, more than $85 \%$ of reported serious adverse events were related to the IDDD, which required surgical revision/removal in half the patients (22).

Recent advances in the field of in vivo gene transfer with adeno-associated virus (AAV) vectors have highlighted the therapeutic potential of this approach, resulting in a therapeutic paradigm that will likely revolutionize the standard of care of inherited diseases. Gene therapy with AAV-derived vectors offers the possibility of lifelong therapeutic benefit following single administrations of gene transfer vectors. Recombinant AAVs stand out for their high transduction efficiency, lack of pathogenicity, and excellent safety record in clinical studies (23-28). AAVs are nonintegrative vectors that persist for years in the nucleus of nondividing cells, predominantly in episomal form $(29,30)$. Studies in large animal models $(24,27,31,32)$, as well as clinical studies in subjects treated with AAVs $(25,26,28,32-37)$, have provided strong evidence of long-term expression for a variety of therapeutic transgenes. Thus, gene therapy can realistically provide a safe alternative to ERT, in particular for diseases such as MPSII, in which the main target organ is the brain, an organ that is difficult to access.

Through delivery of AAV vectors to the CSF, widespread CNS transduction can be achieved with surgical procedures that are common practice in pediatric neurosurgery (38). AAV serotype 9 (AAV9) has unique properties in this regard; not only does AAV9 distribute in much larger areas of the CNS after intraCSF administration compared with other serotypes, but it also reaches the circulation and transduces the liver, providing a peripheral source of therapeutic protein (38-40). We and others have previously demonstrated that intra-CSF delivery of AAV9 vectors is feasible and safe in large animal models, such as dogs and nonhuman primates (38-42). We were also the first to report full disease correction in an animal model of LSD with severe neurologic involvement using this approach (38). However, success in treating one condition does not guarantee therapeutic efficacy of the approach in all other forms of LSD that affect the CNS and peripheral tissues, even if they share common physiopathological mechanisms and similar clinical manifestations.

Here, we describe a therapeutic option for MPSII based on CNS-directed AAV9-mediated gene therapy. Our work provides strong evidence supporting the clinical development of the approach for the treatment of Hunter syndrome patients with cognitive impairment.

\section{Results}

MPSII mice mimic Hunter pathology at 2 months of age. The MPSII mouse model used in this work was obtained by targeted disruption of exons $2-5$ of the murine $I d s$ gene. There was no phenotypic description available for this animal model, so we first set out to characterize the effects of $I d s$ deletion on brain and somatic organs of 2-month-old MPSII males. As expected, IDS activity was almost undetectable throughout the encephalon (Supplemental Figure 1A; supplemental material available online with this article; doi:10.1172/jci.insight.86696DS1); the low levels of activity $(<0.8 \%)$ measured in knockout animals corresponded to background signal of the assay (43). Due to IDS deficiency, all areas of the encephalon presented increased GAG content, which ranged from $121 \%$ to $150 \%$ of WT values (Supplemental Figure 1B). At a histological level, the pathological accumulation of GAGs translated into an increase in the number 


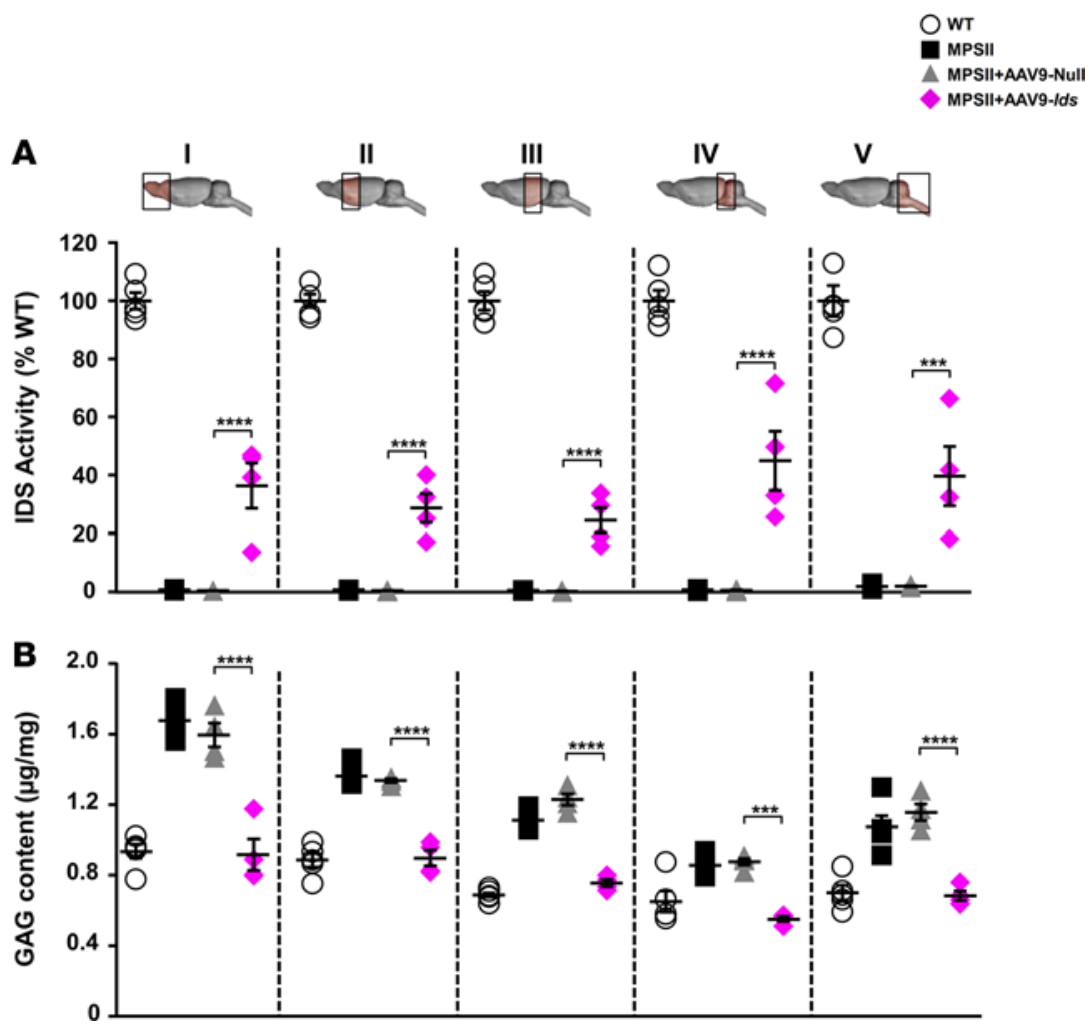

Figure 1. IDS activity and GAG content in the CNS following intra-CSF delivery of AAV9 vectors. (A) Analysis of iduronate-2-sulfatase (IDS) activity in different regions of the encephalon of healthy WT, untreated mucopolysaccharidosis type II (MPSII), and MPSII mice injected in the cerebrospinal fluid (CSF) either with $5 \times$ $10^{10} \mathrm{vg} /$ mouse of null vector (MPSII+AAV9-Null) or an equivalent dose of therapeutic vector (MPSII+AAVg-Ids). Four months after vector delivery (i.e., at 6 months of age) the encephalon of each animal was dissected and sectioned into 5 coronal sections (I-V), as indicated in the diagram above the plot. IDS activity of WT mice was set to $100 \%$. Administration of AAVg-Ids restored IDS activity throughout the encephalon of treated MPSII mice. (B) Quantification of glycosaminoglycan (GAG) content in the same cohort of animals. AAV9-Ids delivery into the CSF resulted in full correction of GAG accumulation in all areas analyzed. Data are shown as mean \pm SEM of $4-5$ animals/group. ${ }^{* * *} P<0.001,{ }^{* * *} P$ $<0.0001$ vs. MPSII+AAV9-Null (Dunnett's test).

and/or size of lysosomes in different areas of the encephalon (Supplemental Figure 1C), as indicated by immunostaining of tissue sections for lysosomal-associated membrane protein 2 (LAMP2).

A common observation in LSD is the change in activity of lysosomal enzymes different from the one affected by the mutation (44-46). Therefore, the activities of N-sulphoglucosamine sulphohydrolase (SGSH), heparan- $\alpha$-glucosaminide $\mathrm{N}$-acetyltransferase (HGSNAT), galactosamine ( $\mathrm{N}$-acetyl)-6-sulfatase (GALNS), and $\beta$-hexosaminidase ( $\beta$-HEXO) were measured in brain extracts. In most cases, enzymatic activities were significantly altered; while HGSNAT and $\beta$-HEXO showed increased activity, the activities of SGSH and GALNS were reduced (Supplemental Figure 1D).

MPSII, like many other LSD with CNS involvement, is characterized by the presence of neuroinflammation, defined by chronic activation of glial cells $(47,48)$. Immunostaining for glial fibrillary acidic protein (GFAP), a protein upregulated upon astrocytic activation (49), and staining with Bandeiraea simplicifolia isolectin B4 (BSI-B4) lectin, which labels microglia (50), revealed the presence of astrocytosis (Supplemental Figure 2A) and microgliosis (Supplemental Figure 2B) in the encephalon of 2-month-old MPSII mice.

At a somatic level, IDS activity was almost undetectable in liver and serum (Supplemental Figure 3A), resulting in pathological GAG accumulation in several somatic tissues (Supplemental Figure 3B). The most noticeable increase was observed in the liver, in which GAG content reached almost 10-fold higher levels than that in WT littermates. Hepatomegaly is a common finding in Hunter syndrome patients (1, $2,51)$, therefore we examined livers for their weights. We found that excessive GAG storage resulted in a slight increase in liver weight in MPSII mice (Supplemental Figure 3C). In addition, the expansion of the lysosomal compartment was easily detectable in different somatic tissues through LAMP1 staining (Supplemental Figure 3D). Similar to brain, the activity of several lysosomal enzymes was altered in liver extracts; in the case of liver, however, the activities of $\alpha$-L-iduronidase (IDUA), $\alpha$ - $\mathrm{N}$-acetylglucosaminidase (NAGLU), and $\beta$-glucuronidase (GUSB) were also considerably affected (Supplemental Figure 3E).

Altogether, these results show that, in this animal model of MPSII disease, pathology is well established at the age of 2 months and resembles human Hunter syndrome.

Intra-CSF delivery of AAV9 vectors to restore IDS activity in CNS. To test the efficacy of intra-CSF delivery of IDS-encoding vectors in treating MPSII disease, AAV9 vectors containing the murine Ids coding sequence under the control of the CAG ubiquitous promoter (AAV9-Ids) were generated. Vectors were administered to the CSF of 2-month-old MPSII males through intracisternal injection at a dose of $5 \times 10^{10}$ 
A

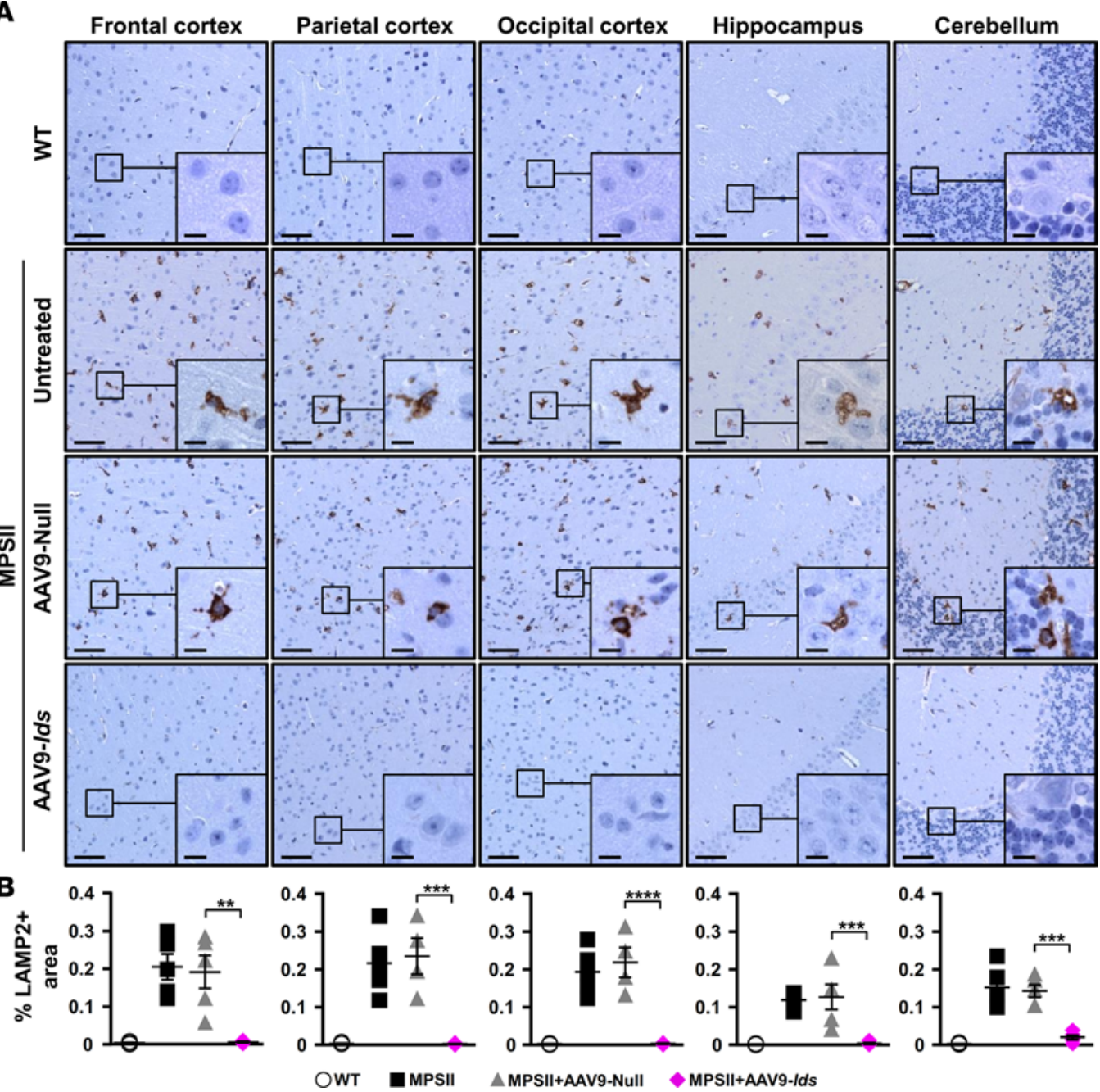

Figure 2. Correction of lysosomal distention in the CNS of MSPII mice by intra-CSF AAV9-Ids delivery. Immunostaining for the lysosomal marker lysosomal-associated membrane protein 2 (LAMP2) to illustrate the size of the lysosomal compartment in different brain regions from 6-month-old healthy WT, untreated mucopolysaccharidosis type II (MPSII), and MPSII mice administered in the cerebrospinal fluid (CSF) with null (MPSII+AAV9-Null) or iduronate-2sulfatase-encoding AAV9 vectors (MPSII+AAV9-Ids). (A) Representative photomicrographs of LAMP2 immunohistochemistry in different brain areas. Scale bar: $50 \mu \mathrm{m} ; 10 \mu \mathrm{m}$ (insets). (B) Histograms represent the quantification of the percentage of LAMP2 + area in each brain region. Data are shown as mean \pm SEM of $4-5$ animals/group. ${ }^{* *} P<0.01$, ${ }^{* *} P<0.001,{ }^{* * * *} P<0.0001$ vs. MPSII+AAV9-Null (Dunnett's test).

vg. Untreated age-matched MPSII mice or those administered with the same dose of a noncoding vector (AAV9-Null) and WT littermates were used as controls. Four months after vector administration (i.e., at 6 months of age), animals were sacrificed and tissues were harvested and processed. While IDS activity remained undetectable in untreated or null-injected MPSII mice, intra-CSF delivery of AAV9-Ids to MPSII mice resulted in restoration of IDS activity in all regions of the encephalon analyzed (sections I-V, Figure 1A), reaching levels of approximately $40 \%$ of healthy WT mice. This observation was consistent with the biodistribution of AAV9-Ids vector genomes and the profile of vector-derived IDS expression documented in the encephalon, which confirmed that after intra-CSF delivery vectors spread throughout the CNS (Supplemental Figure 4, A and B). Colocalization studies using specific cell markers on brain sections from MPSII mice injected in the CSF with AAV9 vectors encoding for the reporter GFP (AAV9-GFP) confirmed our previous observations that the majority of transduced cells were neurons, with much less frequent transduction of astrocytes and no transgene detection in microglia (Supplemental Figure 5).

As a consequence of increased IDS activity, GAG content was completely normalized in all areas of the encephalon in AAV9-Ids-treated animals (Figure 1B). Null-injected MPSII mice showed, however, the 
A

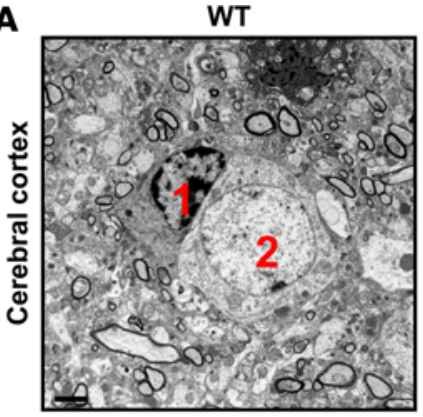

MPSII+AAV9-Null

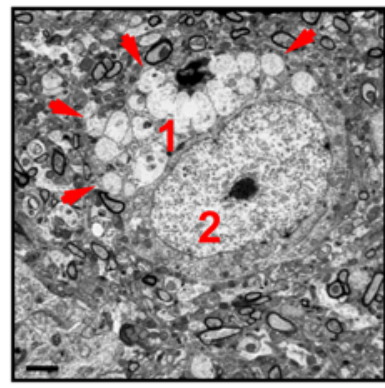

MPSII+AAV9-Ids

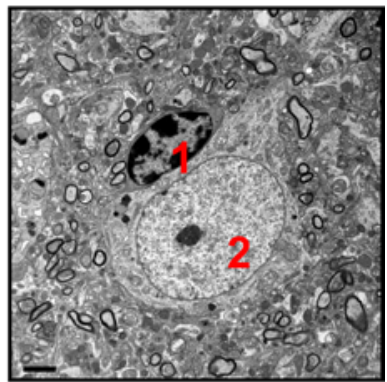

\section{B} Lysosomal activities in Brain

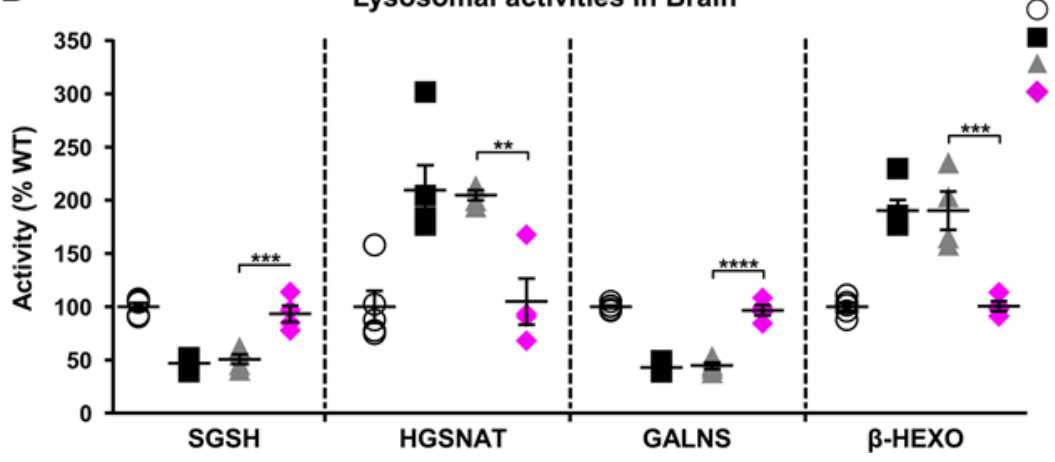

Figure 3. Normalization of lysosomal homeostasis in the brain of MPSII mice following treatment with AAV9-Ids. (A) Representative images of the ultrastructural analysis of the cerebral cortex of WT and mucopolysaccharidosis type II (MPSII) mice performed 4 months after delivery of AAV9-Null or AAV9-Ids vectors to the cerebrospinal fluid. The perineuronal glial cells (noted as 1 ) associated with neurons (noted as 2) of the cerebral cortex of mice treated with AAV9-Ids did not show any of the large electrolucent vacuoles that could be clearly observed in the cytoplasm (indicated by red arrows) of cortical perineuronal glia in MPSII mice that received null vectors. $n=3$. Scale bar: $2 \mu \mathrm{m}$. (B) Four months after gene transfer, the activity of lysosomal enzymes other than iduronate-2-sulfatase was analyzed in brain tissue extracts from all cohorts. WT activity was set to $100 \%$. Treatment with AAVg-Ids restored the activities of N-sulphoglucosamine sulphohydrolase (SCSH), heparan- $\alpha$-glucosaminide $\mathrm{N}$-acetyltransferase (HCSNAT), galactosamine ( $\mathrm{N}$-acetyl)-6-sulfatase (GALNS), and $\beta$-hexosaminidase ( $\beta$-HEXO) in the brain of treated MPSII mice. Data are shown as mean \pm SEM of $4-5$ animals/group. ${ }^{* *} P<0.01,{ }^{* *} P<0.001,{ }^{* * *} P<0.0001$ vs. MPSII+AAV9-Null (Dunnett's test).

same degree of GAG accumulation as untreated MPSII mice (Figure 1B). In agreement with full correction of storage disease, different parts of the encephalon showed equivalent LAMP2+ signal intensity in AAV9-Ids-treated MPSII mice and healthy counterparts (Figure 2). Moreover, the effect of the treatment on lysosomal distension remained stable 8 months after AAV9-Ids delivery (Supplemental Figure 6).

We and others have shown that in animal models of MPSIII disease the heaviest burden of GAG storage in the cerebral cortex is borne by microglial cells $(38,40,52,53)$. By transmission electron microscopy, cells of this type with a cytoplasm full of large electrolucent vacuoles loaded with material — which likely correspond to lysosomes full of undegraded GAGs - can be observed juxtaposed by cortical neurons that have hardly any signs of pathological storage $(38,40,54,55)$. The ultrastructural analysis of the cortex of null-injected 6-month-old MPSII males revealed the same finding (Figure 3A). In contrast, in the cortex of MPSII mice treated with AAV9-Ids, perineuronal glial cells had an appearance similar to that of WT perineuronal glial cells, with no discernible storage vesicles in their cytoplasm (Figure 3A), suggesting complete clearance of accumulated GAGs.

The efficacy of AAV9-Ids treatment was further confirmed by analyzing several lysosomal hydrolases in brain extracts. Untreated and null-injected MPSII mice showed significant increases in HGSNAT and $\beta$-HEXO activity at 6 months of age, whereas SGSH and GALNS activity was reduced. Four months after AAV9-Ids delivery, the activity of all these hydrolases was completely normalized in brains of treated MPSII males (Figure 3B).

Intra-CSF gene therapy corrects neuroinflammation. At 6 months of age, the encephalon of untreated and null-injected MPSII mice showed intense GFAP signal in all regions analyzed, demonstrating marked 
A

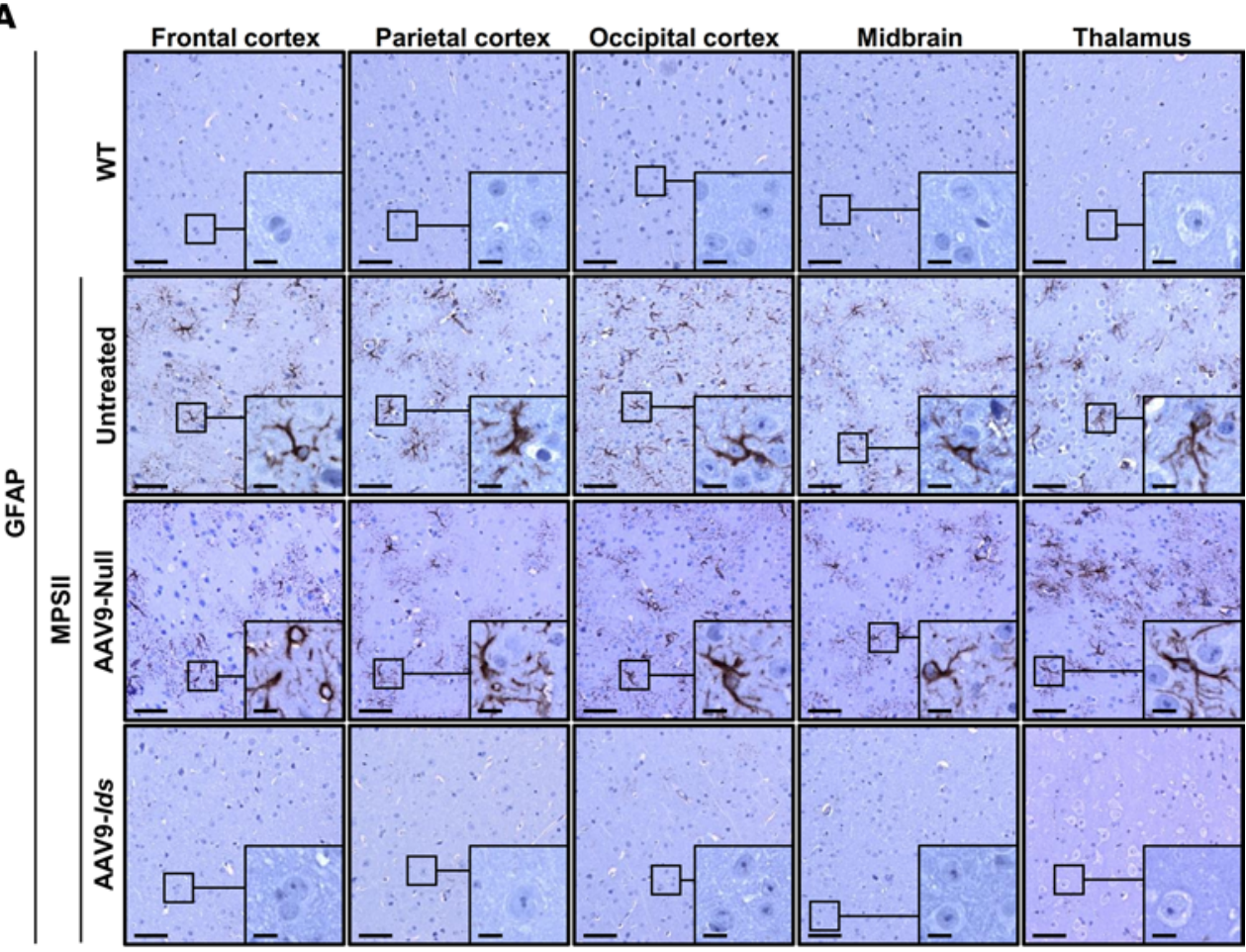

B

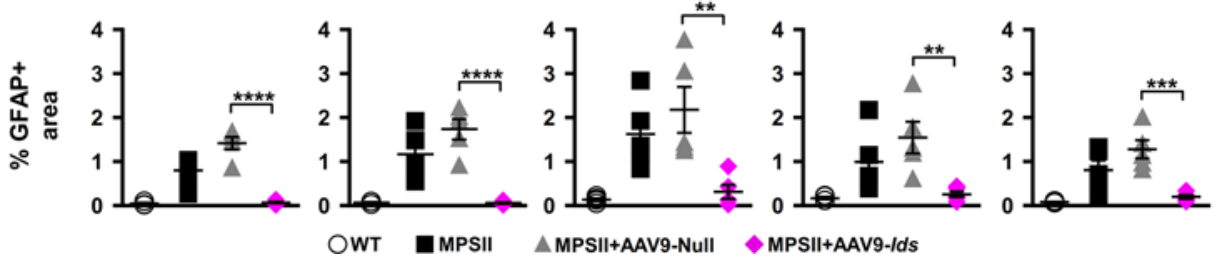

Figure 4. Intra-CSF gene therapy with AAV9-Ids corrects astrocytosis in MPSII mice. Study of the effects of the treatment on astrocytosis at 6 months of age in the brains of healthy WT, untreated mucopolysaccharidosis type II (MPSII), or MPSII mice injected in the cerebrospinal fluid with either null vector (MPSII+AAV9-Null) or therapeutic vector (MPSII+AAVg-Ids). (A) Astrocytosis was evaluated through immunohistochemistry with anti-glial fibrillary acidic protein (anti-GFAP) antibody. Scale bar: $50 \mu \mathrm{m} ; 10 \mu \mathrm{m}$ (insets). (B) The percentage of GFAP-positive area quantified in each brain region is represented in the histograms. Data are shown as mean \pm SEM of $4-5$ animals/ group. ${ }^{* *} P<0.01,{ }^{* *} P<0.001,{ }^{* * *} P<0.0001$ vs. MPSII+AAV9-Null (Dunnett's test).

activation of astrocytes (Figure 4A). GFAP upregulation was absent from the encephalon of mice treated with AAV9-Ids, with detection of scarce, weakly positive astrocytes (Figure 4). The quantification of signal intensity clearly demonstrated upregulation of GFAP in MPSII mice injected with nontherapeutic vectors, and reversion was mediated by AAV9-Ids treatment. A similar observation was made following labeling with BSI-B4, a lectin that, under our staining protocol, preferentially labels activated microglia $(38,40,50,52,54)$. All the encephalon regions analyzed in untreated and null-injected MPSII mice showed a considerable number of vacuolated cells strongly positive for BSI-B4 (Figure 5). Four months after vector delivery, BSI-B4 signal had disappeared from the encephalon of AAV9-Ids-treated MPSII mice (Figure 5). Furthermore, at 10 months of age, the impact of AAV9-Ids treatment on neuroinflammation, evaluated through both markers, was sustained (Supplemental Figure 7, A and B). Altogether, these results indicate that intra-CSF administration of AAV9-Ids in animals with established disease can lead to full and long-term eradication of neuroinflammation.

Recovery of brain transcriptional signature following intra-CSF AAV9-Ids gene therapy. In a previous study we demonstrated correspondence between CNS therapeutic efficacy and normalization of the transcriptomic profile following CNS-directed gene therapy (40). When RNA isolated from the encephalon of WT, AAV9-Ids-, or null-treated MPSII mice was analyzed using the Affymetrix microarray platform, 90 genes were found to be differentially expressed among the 3 groups after data processing. The complete list of 
A

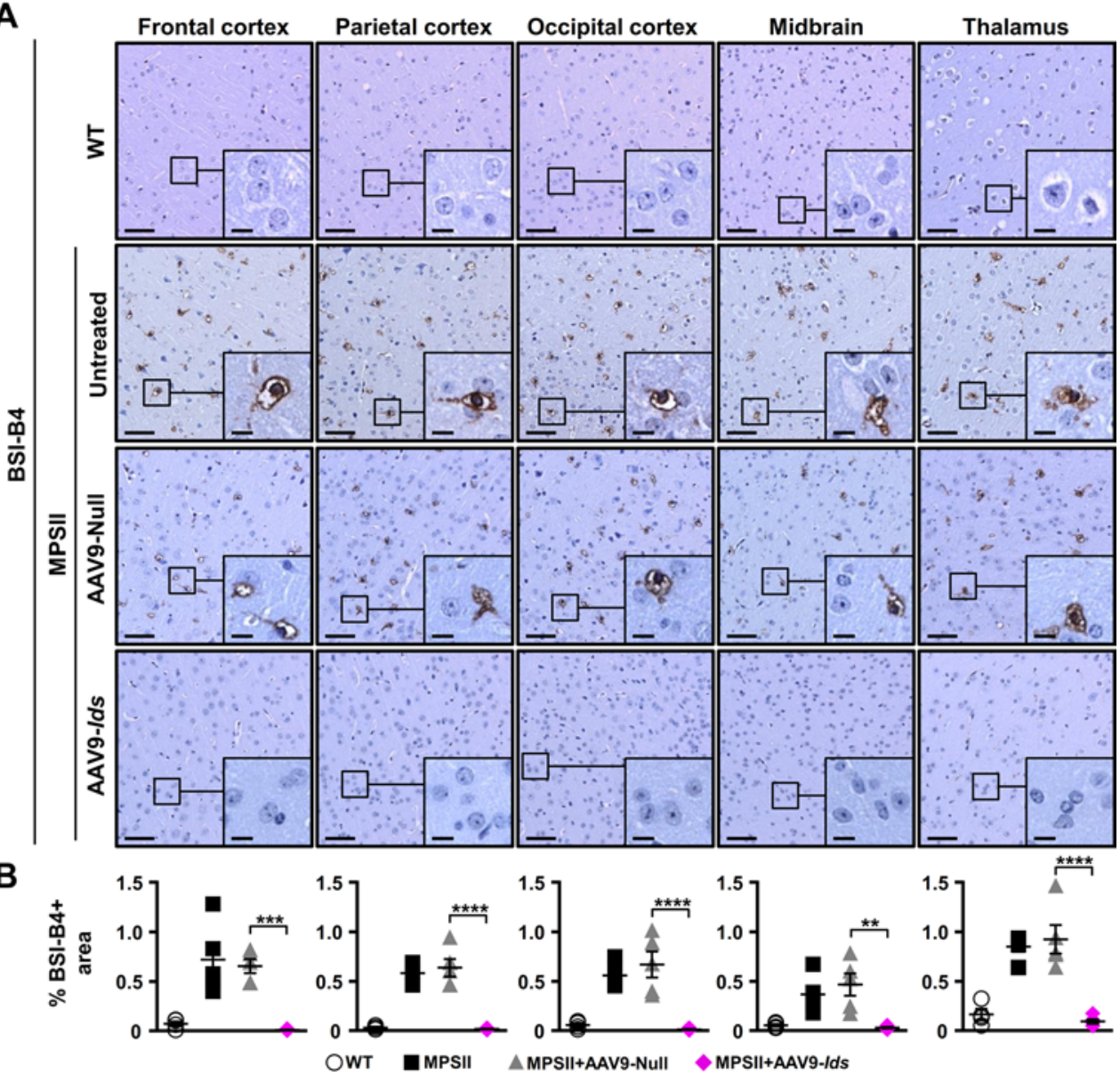

Figure 5. Intra-CSF gene therapy with AAV9-Ids corrects microgliosis in MPSII mice. Study of the effects of the treatment on microgliosis at 6 months of age in the brains of healthy WT, untreated mucopolysaccharidosis type II (MPSII), or MPSII mice injected in the cerebrospinal fluid (CSF) with either null vector (MPSII+AAV9-Null) or therapeutic vector (MPSII+AAV9-Ids). (A) Staining of brain sections for the microglial marker Bandeiraea simplicifolia isolectin B4 (BSI-B4). Representative micrographs are shown. Scale bar: $50 \mu \mathrm{m} ; 10 \mu \mathrm{m}$ (insets). (B) The percentage of BSI-B4-positive area quantified in each brain region is represented in the histograms. Data are shown as mean \pm SEM of 4-5 animals/group. ${ }^{* *} P<0.01,{ }^{* *} P<0.001,{ }^{* * *} P<0.0001$ vs. MPSII+AAVg-Null (Dunnett's test).

genes and their fold change with respect to WT is provided in Supplemental Table 1. Four months after vector administration, MPSII mice treated with AAV9-Ids showed an expression profile in the CNS that resembled that of healthy littermates (Figure 6A). Almost $80 \%$ of the genes differentially expressed in untreated MPSII mice showed a correction in their transcript levels of at least $50 \%$ following AAV9Ids treatment, and in $60 \%$ of them, the correction was of $75 \%$. Functional analysis of the differentially expressed genes showed a clear enrichment in biological processes associated with inflammation and innate immunity (Figure 6B), likely owing to an increased presence of immune cells involved in these processes, such as microglia, in the CNS of untreated MPSII animals. To confirm this hypothesis, we used cell-type enrichment (CTEN) analysis to assess the contribution of different cell types to the observed changes in transcript levels. A clear enrichment score (21.99) was obtained for the microglial population. Moreover, the expression of genes assigned to microglia by CTEN was clearly normalized following treatment with AAV-Ids (Figure 6C). These results are in agreement with our previous observations in MPSIIIB mice (40) and support the established notion that microglia plays a key role in CNS pathology in all forms of MPS $(47,48,53,56,57)$. Moreover, our results demonstrate the efficacy of intra-CSF delivery of AAV9-Ids in eradicating neuroinflammation.

Effects of intra-CSF gene therapy on somatic pathology. The delivery of AAV9 vectors to the CSF resulted in passage of vector to circulation and transduction of peripheral organs, mainly the liver (Supplemental Figure 4). 
A

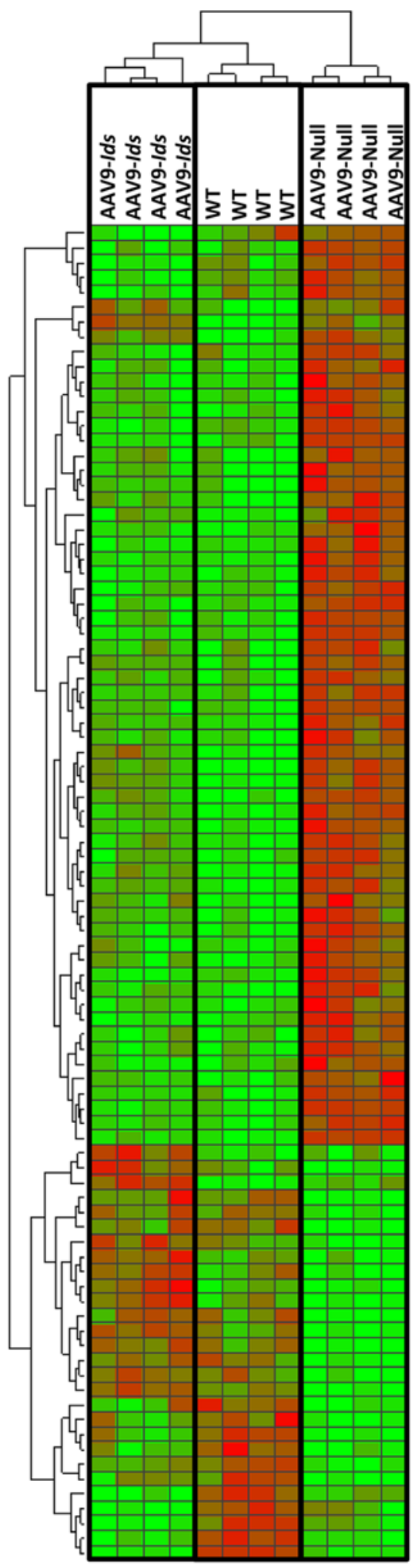

Distance metrics: Euclidean Linkage: COMPLETE
B

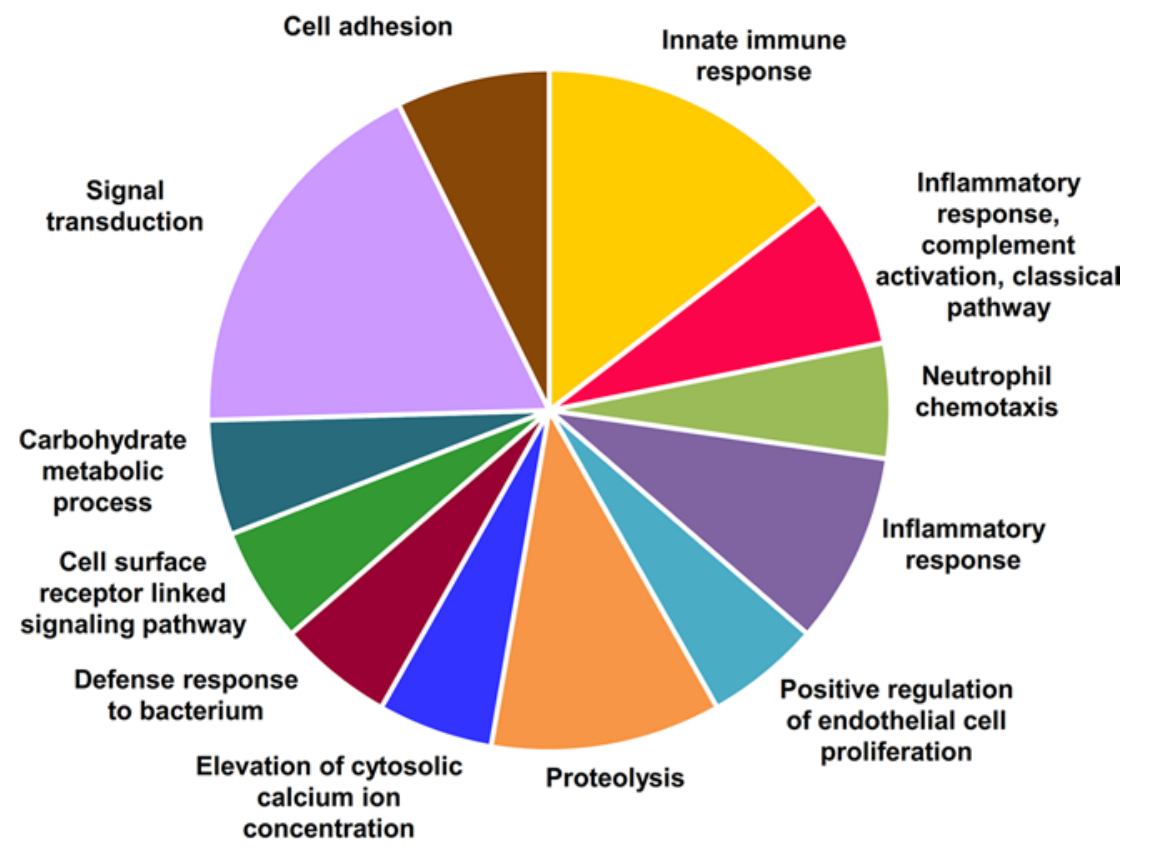

C

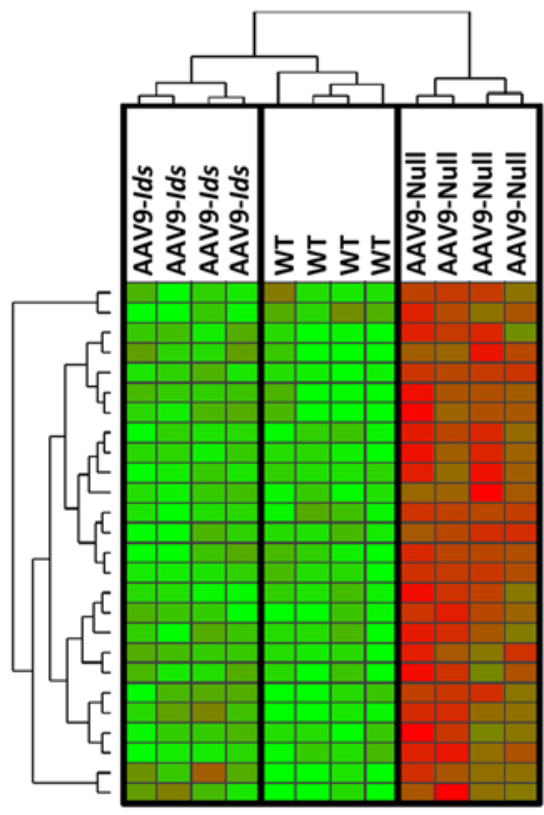

Figure 6. Normalization of CNS transcriptional signature following intra-CSF AAV9-Ids treatment. Microarray expression analysis performed at 6 months of age in WT mice and mucopolysaccharidosis type II (MPSII) littermates 4 months after they received either AAV9-Null or AAV9-Ids vector injections. (A) Hierarchical clustering of all experimental groups based on the complete list of differentially expressed genes. Each row represents a gene and each column represents an animal. The expression of each gene is represented relative to the mean abundance of that gene across all samples in a color scale in which red and green indicate transcript levels above and below the mean, respectively. The magnitude of deviation from the mean is represented by the degree of color saturation. The dendrogram of samples shown above the matrix represents overall similarities in transcript levels. Visibly, the profile of gene expression in MPSII animals treated by intra-cerebrospinal fluid delivery of AAV9-Ids vectors resembles that of healthy age-matched WT littermates. (B) Functional categorization based on Gene Ontology annotation. Most of the categories depicted in the pie chart reflect processes associated with inflammation and innate immunity. (C) Similar analysis by hierarchical clustering as in $\mathbf{A}$ of the set of genes that the cell-type enrichment software assigned to be representative of microglia. 

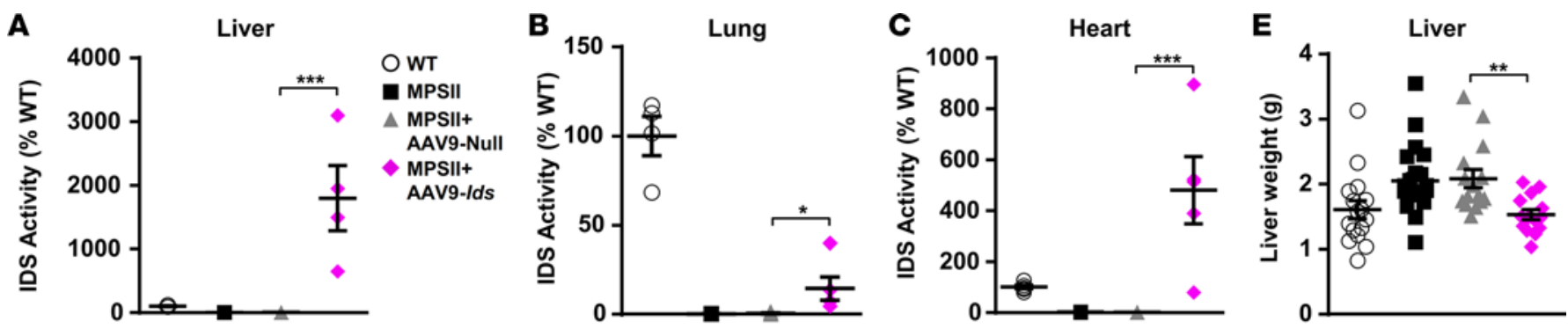
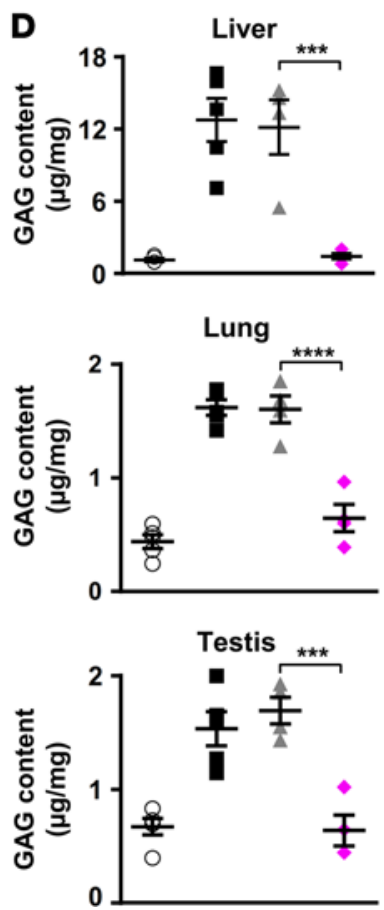
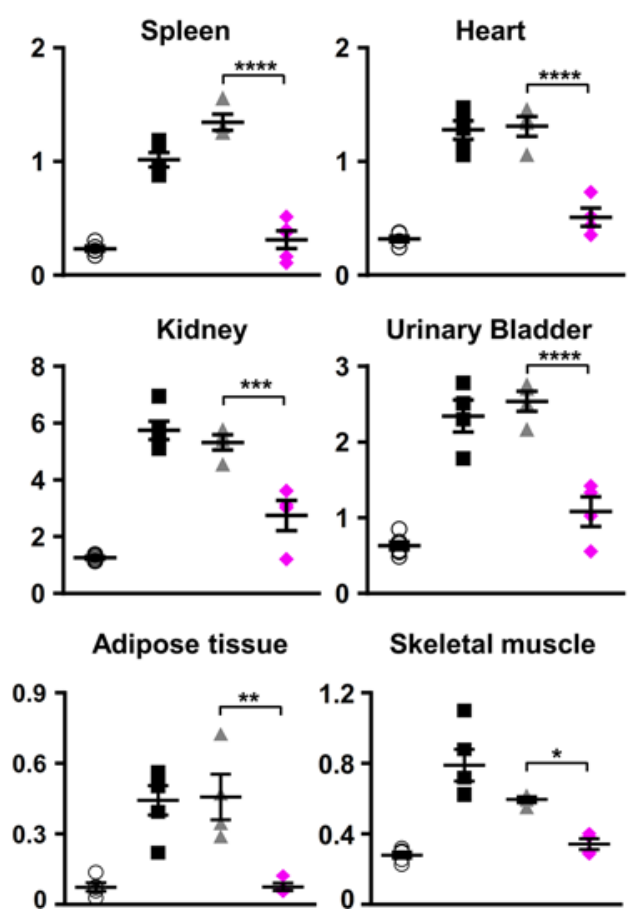

$\mathbf{F}$

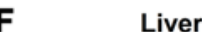

Lung

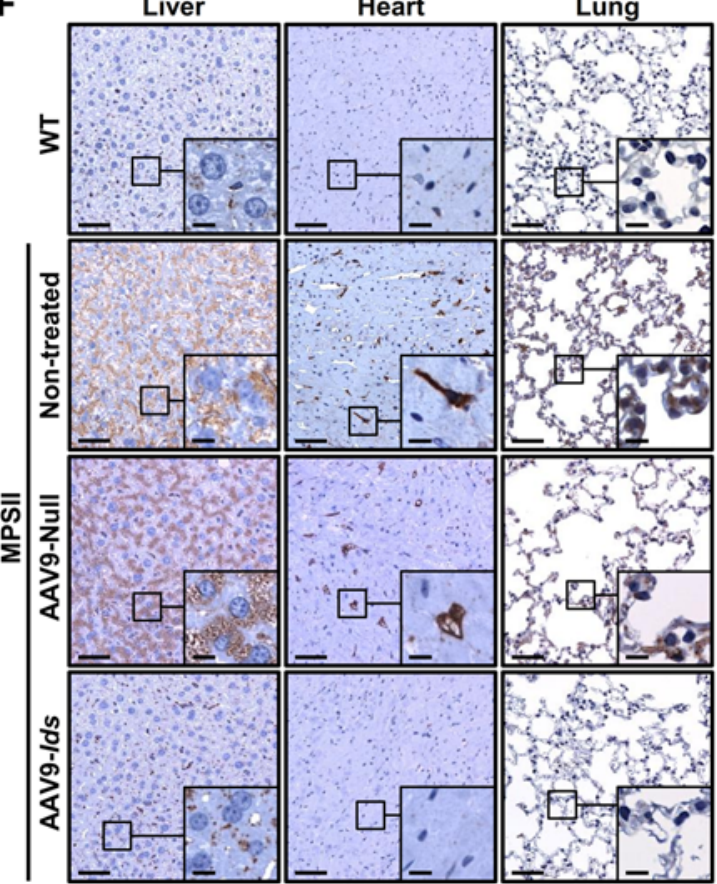

Figure 7. Impact of CSF delivery of Ids-encoding vectors on MPSII somatic pathology. (A-C) Measurement of iduronate-2-sulfatase (IDS) activity in (A) liver, (B) lung, and (C) heart samples obtained from WT, untreated mucopolysaccharidosis type II (MPSII), and MPSII mice injected in the intra-CSF with null vector (MPSII+AAV9-Null) or therapeutic vector (MPSII+AAV9-Ids). IDS activity is expressed as a percentage of WT, where WT activity was set to $100 \%$. (D) Quantification of glycosaminoglycan (GAC) content in somatic organs 4 months after vector delivery. Intra-CSF treatment with AAV9-Ids resulted in full correction of GAG accumulation in almost all somatic tissues analyzed. (E) Weight of the liver in 6-month-old animals. (F) Evaluation of the size of the lysosomal compartment in peripheral organs through lysosomal-associated membrane protein 1 (LAMP1) immunostaining in the same animals. Scale bar: $50 \mu \mathrm{m} ; 10 \mu \mathrm{m}$ (insets). Data are shown as mean \pm SEM of $4-5$ animals/group in $(\mathbf{A}-\mathbf{D}$ and $\mathbf{F})$ and $14-22$ in $(\mathbf{E})$. ${ }^{*} P<0.05,{ }^{*} P<0.01$, ${ }^{* *} P<0.001,{ }^{* * *} P<0.0001$ vs. MPSII+AAVg-Null (Dunnett's test). In B and C, the WT group was excluded from the ANOVA analysis.

Four months following intra-CSF administration of AAV9-Ids to 2-month-old MPSII males, IDS activity was noticeably increased in liver (Figure 7A) and serum (755.9 $\pm 696.5, n=5$ /group). IDS activity was also increased in lung (Figure 7B) and was particularly high in heart (Figure 7C), two organs in which the vector genome copy number/diploid genome was very low $(0.0039 \pm 0.0004$ and $0.0061 \pm 0.0004$ for lung and heart, respectively, $n=5$ /group). Transgene expression was absent (Supplemental Figure 4B), indicating lack of efficient transduction of these organs following intra-CSF AAV9 delivery at the doses used in this study. This finding suggested cross-correction of IDS deficiency by uptake of IDS from circulation. The circulating levels of IDS were sufficient to greatly reduce and, in the cases of liver, spleen, heart, lung, testis, adipose tissue, and skeletal muscle completely correct pathological GAG storage in treated MPSII mice (Figure 7D). Consistent with normalization of GAG content, liver weight was normalized in MPSII mice receiving AAV9Ids vectors (Figure 7E), and immunohistochemistry for LAMP1 revealed a marked reduction in the size of the lysosomal compartment in peripheral organs of MPSII+AAV9-Ids-treated mice (Figure 7F).

Ultrastructural analysis of several somatic tissues at 6 months of age confirmed the efficacy of intra-CSF delivery of AAV9-Ids in treating somatic pathology. A large number of electrolucent vacuoles were visible within hepatocytes of null-injected MPSII mice (Figure 8A). These storage vesicles 
A

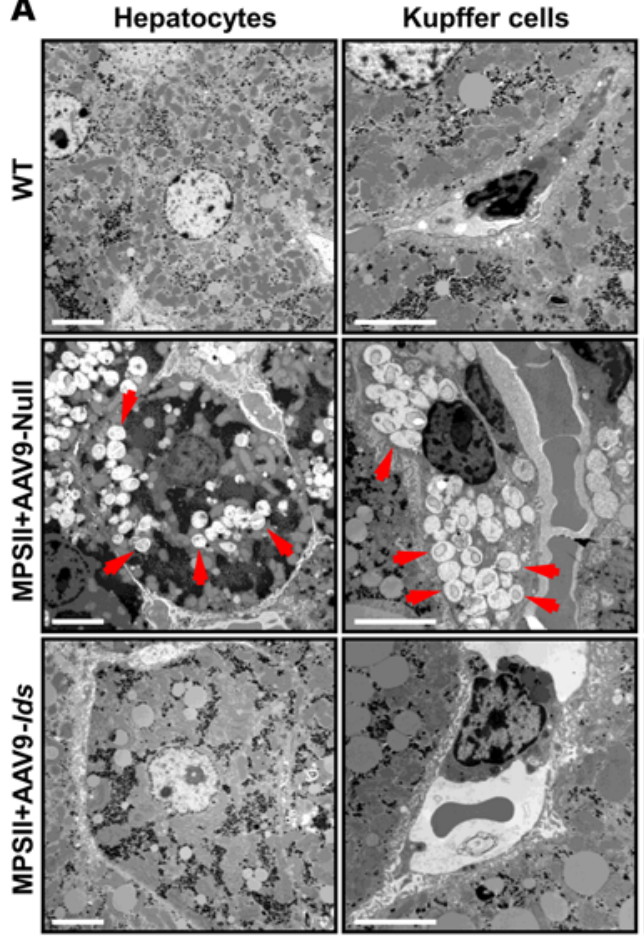

B

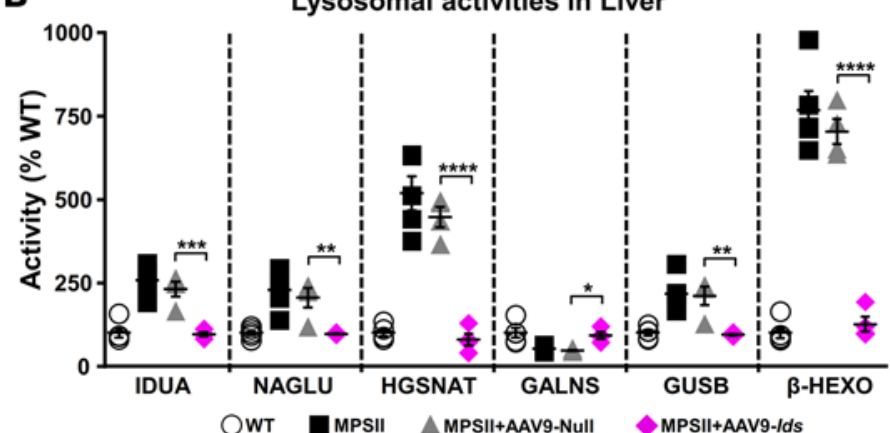

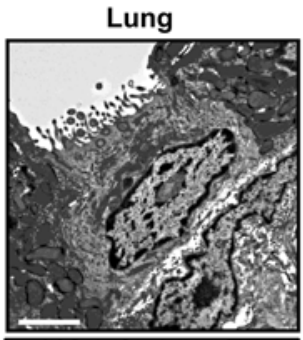
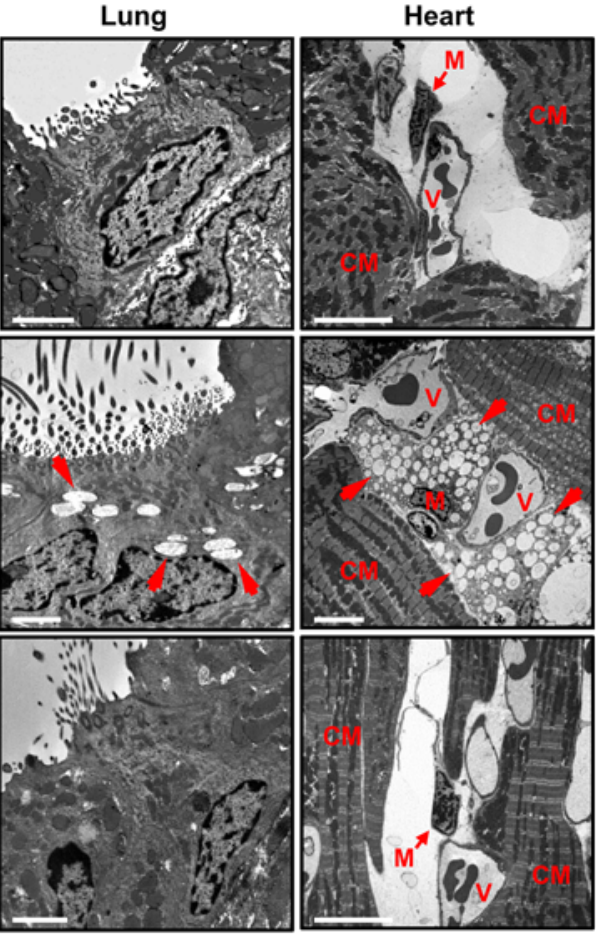

C

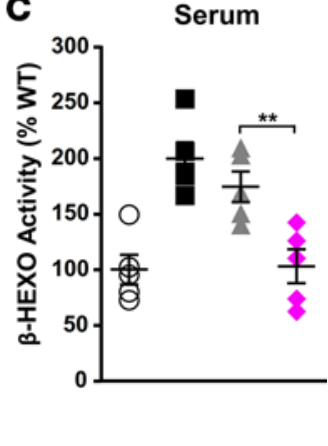

Figure 8. AAV9-Ids mediated correction of lysosomal pathology in somatic organs. (A) Representative images of the ultrastructural analysis of the hepatocytes and Kupffer cells of the liver, lung, and heart performed by transmission electron microscopy 4 months after intra-CSF delivery of vectors. MPSII+AAV9-Null, mucopolysaccharidosis type II (MSPII) mice receiving null vector; MSPII+AAV9-Ids, MPSII mice treated with therapeutic vector. Treated mice showed an evident reduction in the size and number of intracytoplasmic electrolucent vacuoles (red arrows) in all different tissues analyzed. CM, cardiac myocyte; V, blood vessel; M, macrophage-like cell. $n=3$. Scale bar: $5 \mu \mathrm{m}$ for hepatocytes and Kupffer cells; $2 \mu \mathrm{m}$ for lung; $10 \mu \mathrm{m}$ for heart. (B) Four months after gene transfer, the activity of a set of lysosomal enzymes other than IDS was analyzed. WT activity was set to $100 \%$ in all cases. Intra-CSF treatment with AAV9Ids vectors restored the activities of $\alpha$-L-iduronidase (IDUA), $\alpha$ - $N$-acetylglucosaminidase (NAGLU), heparan- $\alpha$-glucosaminide $\mathrm{N}$-acetyltransferase (HCSNAT), galactosamine ( $\mathrm{N}$-acetyl)- 6 -sulfatase (GALNS), $\beta$-glucuronidase (GUSB), and $\beta$-hexosaminidase ( $\beta$-HEXO) in the liver. (C) Intra-CSF AAV9-Ids administration also resulted in correction of $\beta$-HEXO activity in serum. Data are shown as mean \pm SEM of $4-5$ animals/group. ${ }^{*} P<0.05$, ${ }^{* *} P<0.01,{ }^{* * *} P<0.001$, ${ }^{* * * *} P<0.0001$ vs. MPSII+AAV9-Null (Dunnett's test).

probably accumulated undegraded heparan and dermatan sulphate as well as other compounds (58). Larger and more distended storage vesicles were observed in the cytoplasm of Kupffer cells, in ciliated cells of the pulmonary bronchus, and in macrophage-like cells located around blood vessels of the myocardium or interspersed between cardiomyocytes (Figure 8A). In contrast, the cells of the liver, lung, and heart of MPSII mice treated with AAV9-Ids had no storage lesions (Figure 8A) and presented an aspect very similar to that observed in healthy WT animals (Figure 8A).

Similar to the observations made in brain, the activity of several lysosomal hydrolases was altered in the liver of untreated 6-month-old MPSII mice. This lysosomal dysfunction was completely corrected 

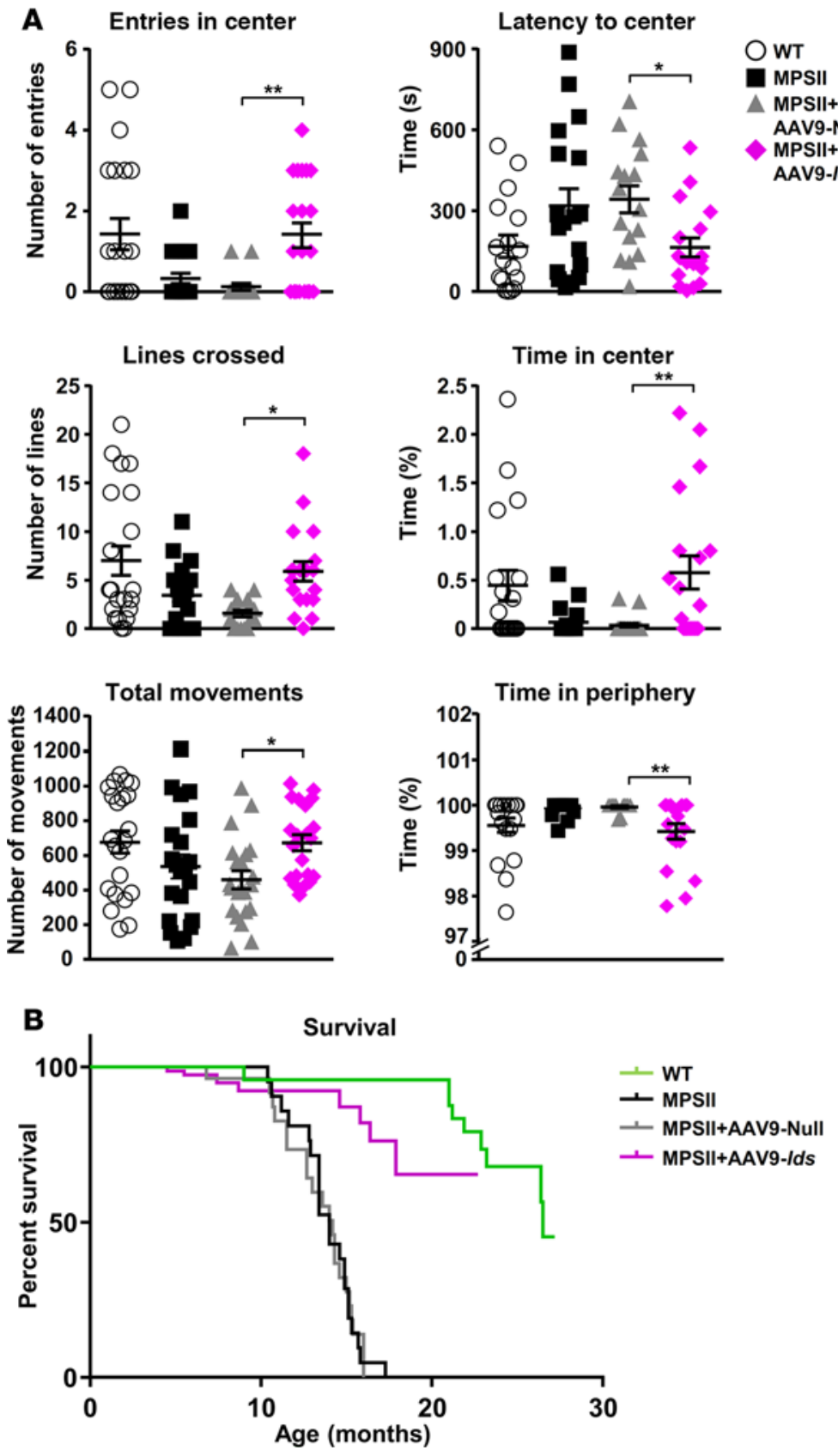

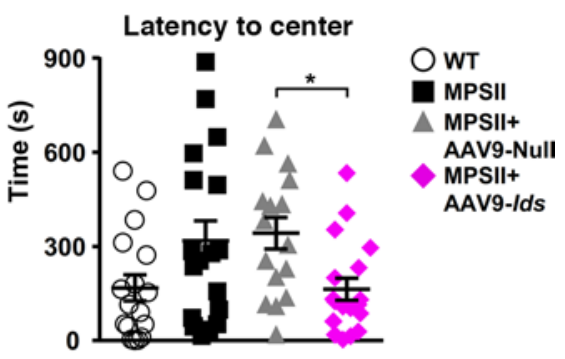

Figure 9. Functional disease correction in MPSII mice treated with intra-CSF gene therapy. (A) Naive-tested WT mice, untreated mucopolysaccharidosis type II (MPSII) mice, and MPSII mice injected in the intra-CSF with $5 \times 10^{10}$ vector genomes (vg) of either null vector (MPSII+AAVg-Null) or therapeutic vector (MSPII+AAV9-Ids) were evaluated by the open-field test 4 months after gene delivery. Treatment with AAV9-Ids resulted in complete correction of the behavioral deficits characteristic of the MPSII mouse model. Data correspond to the locomotor and exploratory activity (entries in center, lines crossed, and total movements) and anxiety (latency to the center, time in center, and time in periphery) recorded during the first 3 minutes and are represented as the mean \pm SEM of $16-22$ animals/group. ${ }^{*} P<0.05,{ }^{*} P<0.01$ vs. MPSII+AAV9Null (Dunnett's test). (B) Kaplan-Meier analysis of survival in the same cohorts of animals as in $\mathbf{A} . n=24$ for WT, 22 for untreated MPSII, 27 for null-injected MPSII, and 91 for mice receiving $5 \times 10^{10} \mathrm{vg}$ of therapeutic AAVg-Ids vector.

following intra-CSF delivery of AAV9-Ids vectors to MPSII mice (Figure 8B) at an age at which lysosomal pathology is already present (Supplemental Figure $3 \mathrm{E})$, providing further evidence of the restoration of lysosomal homeostasis in the liver of treated animals. Furthermore, serum $\beta$-HEXO activity also increased as a consequence of lysosomal pathology, and it was completely normalized following AAV9-Ids treatment (Figure 8C), providing evidence of whole-body correction of lysosomal functionality.

Intra-CSF gene therapy corrects behavioral alterations and prolongs survival of MPSII mice. We used the open-field test, which assesses the general locomotor and exploratory activity of mice in unknown surroundings (59), to evaluate the functional impact of the treatment. The study was performed at 6 months of age, and all animals were naive to the test. Untreated and null-injected MPSII mice displayed reduced locomotor and exploratory activity when compared with age-matched WT animals, as evidenced by the reduced number of entries in the center of the arena, reduced crossing of demarcated lines between the different areas, and reduced total movements (Figure 9A). Untreated animals also showed anxiety-related behavior, as they took longer to enter the center of the arena for the first time and spent less time in the center region

and more time in the periphery (Figure 9A). Intra-CSF delivery of AAV9-Ids vectors completely corrected these behavioral deficits (Figure 9A); there were no statistical differences in any of the parameters analyzed between WT and treated MPSII mice. Finally, intra-CSF gene therapy considerably extended the life span of MPSII mice (Figure 9B). At 17 months of age, all untreated or null-vector treated MPSII mice were dead, while $76 \%$ of MPSII mice receiving AAV9-Ids treatment were alive. Moreover, $65 \%$ of treated animals were still alive at 22 months of age. The percentage of WT animals alive at this age was $79 \%$. Taking into account that MPSII mice already had established disease at the age of treatment, the observation of functional correction in treated animals suggests that it is possible, at least to some extent, to revert the effects of the disease.

\section{Discussion}

The treatment of the neurodegenerative disease that characterizes the most severe and frequent form of MPSII represents a highly unmet medical need and a therapeutic challenge. With the advent of ERT came the possibility of correcting IDS deficiency through administration of recombinant enzyme. However, 
delivery of proteins to the CNS has challenges and shortcomings. In a recent phase I/II clinical trial, monthly intrathecal ERT resulted in drastic and likely clinically meaningful reductions in CSF GAGs, but half of the patients involved in the study required surgical intervention due to problems with the device implanted to provide the enzyme, which included device dislocation, breakage, or malfunction or implant site infection (22). On the other hand, although BMT offers therapeutic benefit to patients affected by other forms of MPS, such as MPSI, it does not improve the neurological outcome of MPSII in patients with severe phenotype (14-16). Here, we report CNS-directed gene therapy developed for the treatment of the severe phenotype of MPSII and provide evidence of the therapeutic efficacy of the approach to treat not only neurologic but also somatic pathology in animals with already established disease.

Previous attempts to treat neurologic MPSII with AAV tested systemic vector delivery to achieve supraphysiological concentrations of circulating IDS $(60,61)$. This strategy was based on earlier work with ERT for different forms of LSD that suggested that when high levels of enzyme are present in the bloodstream, a portion of the protein manages to cross the $\operatorname{BBB}(62,63)$. Cardone et al. combined the capsid of AAV8 - a serotype with high tropism for hepatocytes (64) - and the thyroxin-binding globulin (TBG) promoter to direct the expression of IDS specifically to liver. Following i.v. administration of $4 \times 10^{12} \mathrm{vg} / \mathrm{kg}$ of recombinant AAV8-TBG-IDS vector to adult MPSII mice, plasma concentrations of IDS were 16- to 70-fold higher than in WT, and complete correction of somatic disease was achieved (61). However, efficacy in the brain was limited, with only partial reduction of GAGs observed in this organ, likely due to the fractional amount of IDS that crossed the BBB despite the high concentration of circulating enzyme. We made similar observations in the MPSIIIA mouse model when we directed expression of sulfamidase to the liver (54). Later, the same group tested the i.v. administration of AAV5 vectors to newborn (P2) MPSII mice; IDS expression from this vector was driven by the strong viral promoter CMV (65). Although in this case, the levels of circulating and brain IDS were much lower than those observed in their previous work, reaching only $1 \%$ to $2 \%$ of WT IDS activity in the brain, and the impact on neurological disease seemed to be greater (60), likely due to the treatment of animals at an age at which the disease had barely manifested. It is worth noticing that this therapeutic effect was obtained using very high vector doses $\left(5 \times 10^{13} \mathrm{vg} / \mathrm{kg}\right.$, considering an average weight of $2 \mathrm{~g}$ for a P2 mouse). Using a dose 25 -fold lower $\left(5 \times 10^{10} \mathrm{vg} /\right.$ mouse or $\left.2 \times 10^{12} \mathrm{vg} / \mathrm{kg}\right)$ delivered directly to the CNS, we achieved levels of IDS activity in the brain of adult mice that were approximately $40 \%$ of WT levels as well as supraphysiological levels of enzyme in the liver and circulation that allowed wholebody disease correction. Using the lowest possible therapeutic dose is always desirable in gene therapy with AAV vectors, not only because capsid-directed immune responses are dose dependent $(28,66)$, but also because producing such high doses of GMP-quality vectors suitable for human administration represents a manufacturing challenge.

The IDS-deficient mouse model used in the present study had not been phenotypically characterized before, so we analyzed markers of lysosomal pathology in 2-month-old animals. Treatment was planned at this age in order to mimic the situation of a child in whom diagnoses arrives after a few years of life. In brain, there was a clear accumulation of GAGs with distension of the lysosomal compartment and secondary alteration of the activity of several lysosomal hydrolases. A general increase in lysosomal activity is a common observation in many LSDs $(41,40,67)$ and is mediated through transcriptional activation by transcription factor EB (TFEB) - a master regulator of lysosomal biogenesis and function (44) - in response to the accumulation of undegraded substrates (40). Noticeably, in our mice, while the activity of other enzymes was increased, the activity of SGSH and GALNS was inhibited. This behavior is different to what we had previously observed for SGSH in the brain of mice affected by MPSIIIB (40). It has been noted, however, that pathological accumulation of substrates can inhibit certain lysosomal enzymes. For example, in vitro experiments have shown that heparan sulphate can inhibit GALNS (46), a finding that would explain the reduction in GALNS activity observed in the present study and the presence of keratan sulphate - a substrate of GALNS - in the sera of patients affected by several forms of mucopolysaccharidoses (45). Similarly, SGSH could be inhibited by other compounds. Therefore, the final profile of activity of lysosomal hydrolases seems to be a balance between the effects of TFEB activation and the inhibitions mediated by specific compounds. When MPSII mice were analyzed at 6 months of age, i.e., 4 months after administration of AAV9-Ids vectors, the content of GAGs, the general and ultrastructural aspect, and the size and function of brain lysosomes were all corrected in treated animals, but these had worsened in nontreated MPSII animals or those receiving noncoding AAV9-Null vectors. 
Two-month-old untreated MPSII mice also showed pronounced astrocytosis and microgliosis throughout the brain, a hallmark finding in different animal models of mucopolysaccharidoses that present with neurodegeneration $(38,40,52,54,68-70)$ and in Hunter syndrome patients $(47,48)$. The effects of AAV9mediated IDS gene transfer on the transcriptional signature and histological findings associated with microglial activation in 6-month old animals were quite striking and were in agreement with our previous observations in MPSIIIB mice (40). Several lines of evidence underscore the role played by the secondary activation of microglial/astroglial cells on the etiopathology of neurological dysfunction in LSD (47, 48, $53,56,57,71,72)$. The normalization of the markers of neuroinflammation that appear secondary to IDS deficiency provides further evidence of the resolution of storage disease achieved through intra-CSF delivery of AAV9-Ids. Furthermore, the inhibitory effects on neuroinflammation persisted 8 months after gene therapy, providing evidence of long-term therapeutic efficacy after a single dosing with the treatment.

In 2-month-old untreated MPSII mice there was clear evidence of lysosomal pathology in all the somatic organs analyzed, including liver, heart, lung, and spleen. The accumulation of undegraded GAGs was particularly noticeable in liver, which presented increased weight, and a clear deregulation of the activity of lysosomal hydrolases. Similar to the observations made in the CNS, all of these pathological changes were corrected 4 months after the intra-CSF delivery of IDS-encoding AAV9. Moreover, the analysis of lysosomal distention 8 months after delivery of AAV9-Ids vectors showed that the effects of the therapy persist long-term after a single treatment administration. Further evidence of the improvement in the health condition of AAV9-Ids-treated mice was provided by the open-field test, in which treated MPSII mice showed less anxiety and better locomotor performance, and by the evident extension of the life span of treated animals when compared with animals that did not receive the therapeutic vector.

We chose to treat 2-month-old animals and demonstrated established MPSII disease at this age. Given that most of the pathological changes associated with the alteration of the lysosomal system were already present at treatment, our results demonstrate that CNS-directed AAV9-mediated IDS gene transfer can reverse established MPSII pathology, at least to some extent. MPSII is, however, a progressive neurodegenerative disease, and there probably is a therapeutic period of opportunity, after which full reversal of disease will be hard to achieve.

We have previously performed extensive biodistribution studies in mice and dogs and determined that when AAV9 vectors are delivered to the CSF at the range of doses used in the present study, vector genomes and transgene expression are found throughout the CNS and peripheral nervous system (38, 40). Of particular relevance are the results obtained in the dog, an animal with a brain size closer to that of the target pediatric population. In these studies, the encephalon of 7 dogs was sampled in at least 30 fractions, covering the whole-organ volume. Vector genomes and transgene expression were detected in frontal, parietal, temporal and occipital cortexes, olfactory tract, striatum, hippocampus, piriform lobe, diencephalon, mesencephalon, pons, medulla oblongata, vermis, and cerebellum (38). Moreover, vectors were detected in all portions of the spinal cord up to the cauda equina and were present at relatively high copy numbers in trigeminal and cervical, thoracic, and lumbar dorsal root ganglia (38). Regarding cellular tropism, colocalization studies demonstrated that, in both mice and dogs, AAV9 vectors delivered to the CSF transduced primarily neurons, with scarce transduction of astrocytes and no transduction of microglia $(38,40)$. Efficient transduction of ependymocytes and leptomeninges was also observed (38). Importantly, we demonstrated in dogs that intra-CSF AAV9 administration results in high and sustained levels ( $>41$ months with observation ongoing) of lysosomal enzymes in CSF $(38,40)$. As mentioned before, ERT studies have demonstrated that intra-CSF delivery of enzyme results in substantial and potentially meaningful reductions in GAGs in MPSII patients (22). In somatic organs, the vector was primarily found in liver, with low copy numbers detected in other tissues $(38,40)$. The present study confirmed the same pattern of AAV9 vector biodistribution and tropism after CSF delivery to MPSII mice, with preferential transduction of neurons, spread of vectors throughout the CNS, and transduction of the liver. There is considerable variability in the literature regarding the main CNS cell type targeted by AAV9. Several factors have been held accountable for the differences observed, including the route of delivery $(73,74)$, the age at administration (75), the animal species (73, 76-78), the doses used, the promoter included in the expression cassette (74, $79)$, and even the single- or double-stranded nature of the AAV (80). Alternatively, the quality of vector preparations could play an important role in determining vector tropism (81). We used a second-generation optimized $\mathrm{CsCl}$ protocol that results in markedly higher vector purity - comparable to GMP batches including dramatic reductions in the number of empty capsids (82). Better transduction efficiency has been demonstrated for several AAV serotypes purified under this protocol, in multiple tissues and species (82). 
In contrast to diseases in which it is critical to transduce a particular cell type, such as motoneurons in spinal muscular atrophy, LSDs in which the mutated gene encodes for a secreted lysosomal protein benefit from the possibility of cross-correction. We provide strong evidence supporting the occurrence of cross-correction through IDS present in CSF, sera, or enzymes secreted by cells neighboring nontransduced cells. First, the huge load of storage vesicles visible in the cytoplasm of cortical perineuronal glial cells was completely cleared from MPSII mice treated with AAV9-Ids. As this cell type, typically recognized by microglial markers, is not transduced by AAV9, this observation suggests that the enzymatic deficiency in these cells was corrected through uptake of extracellular enzyme. Second, despite the fact that the liver is the only peripheral organ that it is efficiently transduced through this approach (38-40), the content of GAGs was normalized or greatly reduced in all somatic organs analyzed, suggesting efficient uptake of circulating enzyme and its appropriate trafficking to lysosomes. The detection of increased levels of IDS activity in the heart and lung of AAV9-Ids-treated MPSII mice, two organs poorly transduced after intra-CSF delivery of AAV9 vectors at the doses used in this study, supports this notion.

In summary, this work provides evidence of simultaneous and long-term correction of neurological and systemic MPSII disease by CNS-directed gene therapy. Proof of efficacy was obtained through biochemical, histological, ultrastructural, and functional analysis. Furthermore, as animals had established disease at treatment, our results demonstrate that the tissue damage caused by excessive accumulation of undegraded heparan and dermatan sulphate is, to some extent, reversible. The data provided here support the clinical development of this approach for the treatment of boys with MPSII with somatic disease and any degree of CNS involvement.

\section{Methods}

Animals. IDS-deficient mice (129/SvEv-C57BL/6 genetic background) were purchased from Taconic (TF1838). Affected MPSII mice and healthy littermates were bred from unaffected males and heterozygous females. Genotyping was performed by PCR with primers: Fw1: 5'-TTTTGTGTACTCCAACCCCG-3'; RvWt1: 5'-TGTCTCCATAACAGCCCAGG-3'; RvKO2: 5'-GCCCTCACATTGCCAAAAGA-3'. Mice were fed ad libitum with standard diet (Harlan-Teklad) and were maintained on 12-hour-light/dark cycles.

$A A V$ vector production and administration. AAV vectors were generated by cloning the optimized (GeneArt) version of murine Ids (NM_010498.3, http://www.ncbi.nlm.nih.gov) or GFP cDNA under the control of the ubiquitous hybrid promoter CAG (CMV enhancer, chicken $\beta$-actin promoter, chicken $\beta$-actin intron) into AAV backbone plasmids. AAV9 vectors were produced by triple transfection of HEK293 cells (provided by Katherine High, Children's Hospital of Philadelphia, Philadelphia, Pennsylvania, USA) followed by an optimized cesium chloride gradient-based purification that renders vector batches of high purity (82). Vectors were titered by qPCR. Intracisternal administration of vectors was performed as previously described $(38,83)$. Briefly, the skin of the posterior part of the head of anesthetized mice was shaven, a 2-mm rostro-caudal incision was made, and a Hamilton syringe was introduced between the occiput and C1-vertebra (83) to deliver $5 \mu \mathrm{l}$ of vector into the cisterna magna. All mice were dosed with the same number of vector genomes (vg).

Sample collection. At sacrifice, mice were anesthetized by intraperitoneal injection of ketamine (100 mg/ $\mathrm{kg}$ ) and xylazine $(10 \mathrm{mg} / \mathrm{kg})$. Blood was extracted by cardiac puncture, and subsequently animals were transcardially perfused with $12 \mathrm{ml}$ of PBS to remove all traces of circulating IDS. The entire encephalon and multiple somatic tissues were collected and either snap frozen and stored at $-80^{\circ} \mathrm{C}$ or immersed in formalin for subsequent histological analysis.

Activity of lysosomal enzymes. Liver, brain, heart, and lung samples were sonicated in Milli-Q water (Millipore), and protein extracts were obtained. IDS activity was determined as previously described (84). Briefly, $15 \mu \mathrm{g}$ protein or $2 \mu \mathrm{l}$ serum was first incubated with 4-methylumbelliferyl- $\alpha$-L-iduronide-2-sulfate. $\mathrm{Na}_{2}$ (4-MU- $\alpha$ IdoA-2S, Moscerdam) for 4 hours at $37^{\circ} \mathrm{C}$. A second incubation step was performed for 24 hours at $37^{\circ} \mathrm{C}$ after addition of PiCi-buffer $\left(0.2 \mathrm{M} \mathrm{Na}_{2} \mathrm{HPO}_{4} / 0.1 \mathrm{M}\right.$ citric acid buffer, $\mathrm{pH}=4.5,+0.02 \%$ (w/v) Na-azide) and LEBT (lysosomal enzymes purified from bovine testis) solution. The activities of IDUA, SGSH, NAGLU, GUSB, and $\beta$-HEXO were determined using 4-methylumbelliferone-derived fluoregenic substrates as previously described $(38,40)$. HGSNAT activity was determined on $30 \mu \mathrm{g}$ of protein incubated with acetyl-coenzyme A and 4-methylumbelliferyl- $\beta$-D-glucosaminide (MU- $\beta \mathrm{GlcNH}_{2}$, Moscerdam) for 17 hours at $37^{\circ} \mathrm{C}(85)$. GALNS activity was assayed by a 2-step protocol, with a 17-hour incubation step at $37^{\circ} \mathrm{C}$ in which $10 \mu \mathrm{g}$ protein was mixed with 4 -methylumbelliferyl $\beta$-D-galactopyranoside-6- 
sulphate sodium salt (MU- $\beta$ Gal-6S, Toronto Research Chemical). The second step followed after addition of Pi-buffer (0.9 $\mathrm{M} \mathrm{Na}_{2} \mathrm{HPO}_{4} / 0.9 \mathrm{M} \mathrm{NaH}_{2} \mathrm{PO}_{4}$ buffer, $\mathrm{pH}=4.3,+0.02 \%$ (w/v) Na-azide) and $\beta$-galactosidase (Sigma-Aldrich) and incubation of the mix for 2 hours at $37^{\circ} \mathrm{C}(86)$. After stopping reactions by increasing the $\mathrm{pH}$, released fluorescence was measured with a FLx800 fluorimeter (BioTek Instruments). Brain, liver, heart, and lung activity were normalized against total amount of protein, quantified by Bradford assay (Bio-Rad). Serum activity was normalized by volume.

GAG quantification. Tissues were weighed and digested with proteinase $\mathrm{K}$, and extracts were clarified by centrifugation and filtration (Ultrafree MC, Millipore). GAG levels were determined with Blyscan Glycosaminoglycan Assay (Biocolor) using chondroitin 4-sulphate as standard. Results were normalized to wet tissue weight.

Histology and electron microscopy. Tissues were fixed for 12 to 24 hours in formalin, embedded in paraffin, and sectioned. For immunohistochemical detection of LAMP2, paraffin sections were subjected to heat-induced epitope retrieval in citrate buffer, $\mathrm{pH}=6$, and then incubated overnight at $4^{\circ} \mathrm{C}$ with rat anti-LAMP2 (ab13524; Abcam) and subsequently incubated with biotinylated rabbit anti-rat antibody (E0467, Dako). LAMP1, GFAP, BSI-B4, GFP, neuronal nuclei (NeuN), and ionized calcium-binding adapter molecule 1 (Iba1) stainings were performed as previously $(38,40)$. LAMP2, LAMP1, GFAP, and BSI-B4 signals were amplified by incubating sections with ABC-Peroxidase (Thermo Scientific) and visualized using 3,3-diaminobenzidine (Sigma-Aldrich). Hoechst (B2261, Sigma-Aldrich) was used to counterstain nuclei for fluorescent images. Bright-field images were obtained with an Eclipse 90i microscope (Nikon), and fluorescent images were obtained with a confocal microscope (Leyca Microsystems). NIS Elements Advanced Research 2.20 software was used to quantify signal intensity in four $\times 20$ images/ brain region/animal, using identical threshold settings for all animals. The percentage of positive area was calculated, i.e., the area, in pixels, with a positive signal over the total tissue area in the image. Samples for transmission electron microscopy were processed as previously described (54) and analyzed with a Hitachi H-7000 microscope.

Vector biodistribution and IDS expression. Vector genome copy number was quantified as previously described $(38,40)$ using the following primers and probe: forward primer, 5'-CTTGAGCATCTGACTTCTGGCTAAT-3'; reverse primer, 5'-GATTTGCCCTCCCATATGTCC-3'; probe, 5'-CCGAGTGAGAGACACAAAAAATTCCAACAC-3'. Total RNA was isolated from tissues homogenized in TriPure Isolation Reagent (Roche) using an RNeasy Mini Kit (QIAGEN). After cDNA synthesis (Transcriptor First-Strand cDNA Synthesis Kit, Roche), a specific probe and primers for optimized murine Ids - which do not recognize endogenous $I d s$ - were used to perform quantitative PCR with Light Cycler 480 Probes Master (Roche): forward primer, 5'-AGGACCGTGGACTACAGATAC-3'; reverse primer, 5'-TGGATGTCGCTGAAGTTGG-3'; probe, 5'-CAGGAACTCGCTGGGGTCGAATC-3'. Values were normalized to the expression of murine Rplp0: forward primer, 5'-TGACATCGTCTTTAAACCCCG-3'; reverse primer, 5'-TGTCTGCCTCCCACAATGAAG-3'; probe, 5'-TGTCTTCCCTGGGCATCACGTC-3'.

Transcriptomic analysis. Total RNA was isolated from mouse encephalon with mirVana (Ambion) After cDNA synthesis, samples were hybridized in GeneChip Mouse Gene 2.1 ST 16 array plates (Affymetrix) by Progenika Biopharma. Sample processing was performed using Affymetrix-recommended protocols and equipment. Data normalization was performed using the Affymetrix Expression Console tool with the robust multiarray averaging method, and $\log _{2}$-transformed normalized values were obtained. The analysis was focused on known coding sequences, which, after data filtering, resulted in an initial list of 28,427 genes. For differentially expressed genes, the nominal significance level of each univariate test was set to $P \leq 0.001$. For clustering analysis, data were standardized and represented as heatmaps using J-Express Pro (http://jexpress.bioinfo.no/site/). Functional analysis was performed using Genecodis Tool 2.0 (http://genecodis2.dacya.ucm.es/). Array data have been deposited in the ArrayExpress database (https://www.ebi.ac.uk/arrayexpress/; accession code E-MTAB-4728).

Open-field test. Open-field tests were run between 9:00 am and 2:00 pm. Animals were placed in the lower left corner of a brightly lit chamber $(41 \times 41 \times 30 \mathrm{~cm})$ crossed by 2 bundles of photobeams (SedaCom 32; Panlab) that detect horizontal and vertical movements. The area was divided into 3 concentric square regions: center $(14 \times 14 \mathrm{~cm})$, periphery $(27 \times 27 \mathrm{~cm})$, and border $(41 \times 41 \mathrm{~cm})$. Exploratory and motor activities were recorded during the first 3 minutes of the test using a video-tracking system (SmartJunior, Panlab).

Statistics. Results are expressed as mean \pm SEM. Statistical comparisons were made using 2-tailed Student's $t$ test or 1 -way ANOVA. Multiple comparisons were made using Dunnett's post test. $P<0.05$ was 
considered statistically significant. Kaplan-Meier curves were used to estimate survival, and the long-rank test was used for comparisons.

Study approval. All experimental procedures were approved by the Ethics Committee for Animal and Human Experimentation of the Universitat Autònoma de Barcelona.

\section{Author contributions}

FB coordinated the study. S Motas, VH, and FB designed the study and wrote the paper. JR performed the ultrastructural studies. MG performed the transcriptomic analysis. XL produced AAV vectors. S Motas, S Marcó, AR, CR, XS, VS, MM, JB, and LM generated reagents and performed and analyzed experiments. All authors reviewed the results and approved the final version of the manuscript.

\section{Acknowledgments}

We thank Marta Moya, Ángel Vázquez, Verónica Melgarejo, Lorena Noya, and David Ramos for technical assistance. This work was supported by funding from Plan Nacional I+D+i from the Ministerio de Economía y Competitividad (INNPACTO IPT-2012-0772-300000 and SAF 2014-54866-R), Generalitat de Catalunya (2014SGR-1669 and ICREA Academia award to F. Bosch), MPS España Foundation, and the European Union through the European Regional Development Funds. This work is part of a public-private partnership on gene therapy between the Universitat Autònoma de Barcelona and Laboratorios ESTEVE S.A. S. Motas, S. Marcó, and V. Sánchez received a predoctoral fellowship from Generalitat de Catalunya, Spain.

Address correspondence to: Fatima Bosch, Center of Animal Biotechnology and Gene Therapy, Edifici H, Universitat Autònoma de Barcelona, E-08193 Bellaterra, Spain. Phone: 34935814182; E-mail: fatima.bosch@uab.es.

1. Neufeld EF, Muenzer J. The mucopolysaccharidoses. In: Scriver CR, et al., eds. The Metabolic and Molecular Basis of Inherited Disease New York, New York, USA: McGraw-Hill, Medical Publishing Division; 2001:3421-3454.

2. Wraith JE, et al. Mucopolysaccharidosis type II (Hunter syndrome): a clinical review and recommendations for treatment in the era of enzyme replacement therapy. Eur J Pediatr. 2008;167(3):267-277.

3. Muenzer J. Overview of the mucopolysaccharidoses. Rheumatology (Oxford). 2011;50(suppl 5):v4-v12.

4. Okuyama T, et al. Japan Elaprase Treatment (JET) study: idursulfase enzyme replacement therapy in adult patients with attenuated Hunter syndrome (Mucopolysaccharidosis II, MPS II). Mol Genet Metab. 2010;99(1):18-25.

5. Yamada Y, et al. Mucopolysaccharidosis type II (Hunter disease): 13 gene mutations in 52 Japanese patients and carrier detection in four families. Hum Genet. 1993;92(2):110-114.

6. Schwartz IV, et al. A clinical study of 77 patients with mucopolysaccharidosis type II. Acta Paediatr. 2007;96(455):63-70.

7. Martin R, et al. Recognition and diagnosis of mucopolysaccharidosis II (Hunter syndrome). Pediatrics. 2008;121(2):e377-e386.

8. Al Sawaf S, Mayatepek E, Hoffmann B. Neurological findings in Hunter disease: pathology and possible therapeutic effects reviewed. J Inherit Metab Dis. 2008;31(4):473-480.

9. Enns GM, Huhn SL. Central nervous system therapy for lysosomal storage disorders. Neurosurg. 2008;24(3-4):E12.

10. Yamada Y, et al. Treatment of MPS VII (Sly disease) by allogeneic BMT in a female with homozygous A619V mutation. Bone Marrow Transplant. 1998;21(6):629-634.

11. Peters C1, Steward CG; National Marrow Donor Program; International Bone Marrow Transplant Registry, Working Party on Inborn Errors, European Bone Marrow Transplant Group. Hematopoietic cell transplantation for inherited metabolic diseases: an overview of outcomes and practice guidelines. Bone Marrow Transplant. 2003;31(4):229-239.

12. Peters C, et al. Outcome of unrelated donor bone marrow transplantation in 40 children with Hurler syndrome. Blood. 1996;87(11):4894-4902.

13. Podetz-Pedersen KM, et al. The American Society of Gene \& Cell Therapy Abstracts from the 16th Annual Meeting, Salt Lake City, Utha: "Prevention of Neurocognitive Deficit by Ex Vivo Lentiviral Transduction of Hematopoietic Stem Cells in a Murine Model of Mucop. Mol Ther. 2013;21(supplement 1):S191.

14. Prasad VK, Kurtzberg J. Transplant outcomes in mucopolysaccharidoses. Semin Hematol. 2010;47(1):59-69.

15. Seto T, et al. Brain magnetic resonance imaging in 23 patients with mucopolysaccharidoses and the effect of bone marrow transplantation. Ann Neurol. 2001;50(1):79-92.

16. Vellodi A, Young E, Cooper A, Lidchi V, Winchester B, Wraith JE. Long-term follow-up following bone marrow transplantation for Hunter disease. J Inherit Metab Dis. 1999;22(5):638-648.

17. Giugliani R, et al. Mucopolysaccharidosis I, II, and VI: Brief review and guidelines for treatment. Genet Mol Biol. 2010;33(4):589-604.

18. Valayannopoulos V, Wijburg FA. Therapy for the mucopolysaccharidoses. Rheumatology (Oxford). 2011;50(suppl 5):v49-v59.

19. Muenzer J, et al. Long-term, open-labeled extension study of idursulfase in the treatment of Hunter syndrome. Genet Med. 2011;13(2):95-101.

20. Muenzer J, et al. A phase II/III clinical study of enzyme replacement therapy with idursulfase in mucopolysaccharidosis II (Hunter syndrome). Genet Med. 2006;8(8):465-473. 
21. Muenzer J, Gucsavas-Calikoglu M, McCandless SE, Schuetz TJ, Kimura A. A phase I/II clinical trial of enzyme replacement therapy in mucopolysaccharidosis II (Hunter syndrome). Mol Genet Metab. 2007;90(3):329-337.

22. Muenzer J, et al. A phase I/II study of intrathecal idursulfase-IT in children with severe mucopolysaccharidosis II. Genet Med. 2016;18(1):73-81.

23. Bainbridge JW, et al. Effect of gene therapy on visual function in Leber's congenital amaurosis. $N$ Engl J Med. 2008;358(21):2231-2239.

24. Rivera VM, et al. Long-term pharmacologically regulated expression of erythropoietin in primates following AAV-mediated gene transfer. Blood. 2005;105(4):1424-1430.

25. Hauswirth WW, et al. Treatment of leber congenital amaurosis due to RPE65 mutations by ocular subretinal injection of adeno-associated virus gene vector: short-term results of a phase I trial. Hum Gene Ther. 2008;19(10):979-990.

26. Maguire AM, et al. Safety and efficacy of gene transfer for Leber's congenital amaurosis. N Engl J Med. 2008;358(21):22402248.

27. Niemeyer GP, et al. Long-term correction of inhibitor-prone hemophilia B dogs treated with liver-directed AAV2-mediated factor IX gene therapy. Blood. 2009;113(4):797-806

28. Nathwani AC, et al. Long-term safety and efficacy of factor IX gene therapy in hemophilia B. NEngl J Med. 2014;371(21):19942004.

29. Wolf DA, Banerjee S, Hackett PB, Whitley CB, McIvor RS, Low WC. Gene therapy for neurologic manifestations of mucopolysaccharidoses. Expert Opin Drug Deliv. 2015;12(2):283-296.

30. Lisowski L, Tay SS, Alexander IE. Adeno-associated virus serotypes for gene therapeutics. Curr Opin Pharmacol. 2015;24:59-67.

31. Callejas D, et al. Treatment of diabetes and long-term survival after insulin and glucokinase gene therapy. Diabetes. 2013;62(5):1718-1729.

32. Bainbridge JW, et al. Long-term effect of gene therapy on Leber's congenital amaurosis. NEngl J Med. 2015;372(20):1887-1897.

33. Testa F, et al. Three-year follow-up after unilateral subretinal delivery of adeno-associated virus in patients with Leber congenital Amaurosis type 2. Ophthalmology. 2013;120(6):1283-1291.

34. Gaudet D, et al. Efficacy and long-term safety of alipogene tiparvovec (AAV1-LPLS447X) gene therapy for lipoprotein lipase deficiency: an open-label trial. Gene Ther. 2013;20(4):361-369.

35. Jacobson SG, et al. Gene therapy for leber congenital amaurosis caused by RPE65 mutations: safety and efficacy in 15 children and adults followed up to 3 years. Arch Ophthalmol. 2012;130(1):9-24.

36. Buchlis G, et al. Factor IX expression in skeletal muscle of a severe hemophilia B patient 10 years after AAV-mediated gene transfer. Blood. 2012;119(13):3038-3041.

37. Simonelli F, et al. Gene therapy for Leber's congenital amaurosis is safe and effective through 1.5 years after vector administration. Mol Ther. 2010;18(3):643-650.

38. Haurigot V, et al. Whole body correction of mucopolysaccharidosis IIIA by intracerebrospinal fluid gene therapy. J Clin Invest. 2013;123(8):3254-3271.

39. Gray SJ, Nagabhushan Kalburgi S, McCown TJ, Jude Samulski R. Global CNS gene delivery and evasion of anti-AAV-neutralizing antibodies by intrathecal AAV administration in non-human primates. Gene Ther. 2013;20(4):450-459.

40. Ribera A, et al. Biochemical, histological and functional correction of mucopolysaccharidosis type IIIB by intra-cerebrospinal fluid gene therapy. Hum Mol Genet. 2015;24(7):2078-2095.

41. Hinderer C, et al. Neonatal systemic AAV induces tolerance to CNS gene therapy in MPS I dogs and nonhuman primates. Mol Ther. 2015;23(8):1298-1307.

42. Samaranch L, et al. Adeno-associated virus serotype 9 transduction in the central nervous system of nonhuman primates. Hum Gene Ther. 2012;23(4):382-389.

43. Higuchi T, et al. Enzyme replacement therapy (ERT) procedure for mucopolysaccharidosis type II (MPS II) by intraventricular administration (IVA) in murine MPS II. Mol Genet Metab. 2012;107(1-2):122-128.

44. Sardiello M, et al. A gene network regulating lysosomal biogenesis and function. Science. 2009;325(5939):473-477.

45. Tomatsu S, et al. Keratan sulphate levels in mucopolysaccharidoses and mucolipidoses. J Inherit Metab Dis. 2005;28(2):187-202.

46. Rowan DJ, Tomatsu S, Grubb JH, Montaño AM, Sly WS. Assessment of bone dysplasia by micro-CT and glycosaminoglycan levels in mouse models for mucopolysaccharidosis type I, IIIA, IVA, and VII. J Inherit Metab Dis. 2013;36(2):235-246.

47. Hamano K, Hayashi M, Shioda K, Fukatsu R, Mizutani S. Mechanisms of neurodegeneration in mucopolysaccharidoses II and IIIB: analysis of human brain tissue. Acta Neuropathol. 2008;115(5):547-559.

48. Constantopoulos G, Iqbal K, Dekaban AS. Mucopolysaccharidosis types IH, IS, II, and IIIA: glycosaminoglycans and lipids of isolated brain cells and other fractions from autopsied tissues. J Neurochem. 1980;34(6):1399-1411.

49. Eng LF, Ghirnikar RS. GFAP and astrogliosis. Brain Pathol. 1994;4(3):229-237.

50. Streit WJ, Kreutzberg GW. Lectin binding by resting and reactive microglia. J Neurocytol. 1987;16(2):249-260

51. Hunter C. A rare disease in two brothers. Proc R Soc Med. 1917;10(Sect study dis child):104-116.

52. Ruzo A, et al. Correction of pathological accumulation of glycosaminoglycans in central nervous system and peripheral tissues of MPSIIIA mice through systemic AAV9 gene transfer. Hum Gene Ther. 2012;23(12):1237-1246.

53. Ohmi K, Greenberg DS, Rajavel KS, Ryazantsev S, Li HH, Neufeld EF. Activated microglia in cortex of mouse models of mucopolysaccharidoses I and IIIB. Proc Natl Acad Sci U S A. 2003;100(4):1902-1907.

54. Ruzo A, et al. Liver production of sulfamidase reverses peripheral and ameliorates CNS pathology in mucopolysaccharidosis IIIA mice. Mol Ther. 2012;20(2):254-266.

55. Fraldi A, et al. Functional correction of CNS lesions in an MPS-IIIA mouse model by intracerebral AAV-mediated delivery of sulfamidase and SUMF1 genes. Hum Mol Genet. 2007;16(22):2693-2702.

56. DiRosario J, et al. Innate and adaptive immune activation in the brain of MPS IIIB mouse model. J Neurosci Res. 2009;87(4):978-990

57. Archer LD, Langford-Smith KJ, Bigger BW, Fildes JE. Mucopolysaccharide diseases: a complex interplay between neuroinflammation, microglial activation and adaptive immunity. J Inherit Metab Dis. 2014;37(1):1-12.

58. Resnick JM, Whitley CB, Leonard AS, Krivit W, Snover DC. Light and electron microscopic features of the liver in mucopoly- 
saccharidosis. Hum Pathol. 1994;25(3):276-286.

59. Bailey K, Crawley J. Anxiety-related behaviors in mice. In: Buccafusco JJ, ed. Methods of Behavior Analysis in Neuroscience. 2nd ed. Boca Raton, Florida, USA: CRC Press/Taylor \& Francis; 2009:Chapter 5.

60. Polito VA, Cosma MP. IDS crossing of the blood-brain barrier corrects CNS defects in MPSII mice. Am J Hum Genet. 2009;85(2):296-301.

61. Cardone M, et al. Correction of Hunter syndrome in the MPSII mouse model by AAV2/8-mediated gene delivery. Hum Mol Genet. 2006;15(7):1225-1236.

62. Vogler C, et al. Overcoming the blood-brain barrier with high-dose enzyme replacement therapy in murine mucopolysaccharidosis VII. Proc Natl Acad Sci U S A. 2005;102(41):14777-14782.

63. Ou L, Herzog T, Koniar BL, Gunther R, Whitley CB. High-dose enzyme replacement therapy in murine Hurler syndrome. Mol Genet Metab. 2014;111(2):116-122.

64. Wu Z, Asokan A, Samulski RJ. Adeno-associated virus serotypes: vector toolkit for human gene therapy. Mol Ther. 2006;14(3):316-327.

65. Xia W, et al. High levels of protein expression using different mammalian CMV promoters in several cell lines. Protein Expr Purif. 2006;45(1):115-124.

66. Manno CS, et al. Successful transduction of liver in hemophilia by AAV-Factor IX and limitations imposed by the host immune response. Nat Med. 2006;12(3):342-347.

67. Settembre C, Fraldi A, Medina DL, Ballabio A. Signals from the lysosome: a control centre for cellular clearance and energy metabolism. Nat Rev Mol Cell Biol. 2013;14(5):283-296.

68. Richard M, Arfi A, Rhinn H, Gandolphe C, Scherman D. Identification of new markers for neurodegeneration process in the mouse model of Sly disease as revealed by expression profiling of selected genes. J Neurosci Res. 2008;86(15):3285-3294

69. Baldo G, et al. Shotgun proteomics reveals possible mechanisms for cognitive impairment in Mucopolysaccharidosis I mice. Mol Genet Metab. 2015;114(2):138-145.

70. Fu H, Bartz JD, Stephens RL, McCarty DM. Peripheral nervous system neuropathology and progressive sensory impairments in a mouse model of Mucopolysaccharidosis IIIB. PLoS One. 2012;7(9):e45992.

71. Walkley SU. Pathogenic mechanisms in lysosomal disease: a reappraisal of the role of the lysosome. Acta Paediatr. 2007;96(455):26-32.

72. McGeer PL, McGeer EG. Glial cell reactions in neurodegenerative diseases: pathophysiology and therapeutic interventions Alzheimer Dis Assoc Disord. 1998;12(suppl 2):S1-S6.

73. Gurda BL, et al. Evaluation of AAV-mediated gene therapy for central nervous system disease in canine Mucopolysaccharidosis VII. Mol Ther. 2016;24(2):206-216.

74. Snyder BR, et al. Comparison of adeno-associated viral vector serotypes for spinal cord and motor neuron gene delivery. Hum Gene Ther. 2011;22(9):1129-1135.

75. Foust KD, Nurre E, Montgomery CL, Hernandez A, Chan CM, Kaspar BK. Intravascular AAV9 preferentially targets neonatal neurons and adult astrocytes. Nat Biotechnol. 2009;27(1):59-65.

76. Bevan AK, et al. Systemic gene delivery in large species for targeting spinal cord, brain, and peripheral tissues for pediatric disorders. Mol Ther. 2011;19(11):1971-1980.

77. Bucher T, et al. Intracisternal delivery of AAV9 results in oligodendrocyte and motor neuron transduction in the whole central nervous system of cats. Gene Ther. 2014;21(5):522-528.

78. Jackson KL, Dayton RD, Klein RL. AAV9 supports wide-scale transduction of the CNS and TDP-43 disease modeling in adult rats. Mol Ther Methods Clin Dev. 2015;2:15036.

79. Duque S, et al. Intravenous administration of self-complementary AAV9 enables transgene delivery to adult motor neurons. Mol Ther. 2009;17(7):1187-1196.

80. Donsante A, et al. Intracerebroventricular delivery of self-complementary adeno-associated virus serotype 9 to the adult rat brain. Gene Ther. 2016;23(5):401-407.

81. Klein RL, Dayton RD, Tatom JB, Henderson KM, Henning PP. AAV8, 9, Rh10, Rh43 vector gene transfer in the rat brain: effects of serotype, promoter and purification method. Mol Ther. 2008;16(1):89-96.

82. Ayuso E, et al. High AAV vector purity results in serotype- and tissue-independent enhancement of transduction efficiency. Gene Ther. 2010;17(4):503-510.

83. Reijneveld JC, Taphoorn MJ, Voest EE. A simple mouse model for leptomeningeal metastases and repeated intrathecal therapy. J Neurooncol. 1999;42(2):137-142.

84. Voznyi YV, Keulemans JL, van Diggelen OP. A fluorimetric enzyme assay for the diagnosis of MPS II (Hunter disease). $J$ Inherit Metab Dis. 2001;24(6):675-680.

85. Voznyi YaV, et al. A fluorimetric enzyme assay for the diagnosis of Sanfilippo disease C (MPS III C). J Inherit Metab Dis. $1993 ; 16(2): 465-472$

86. van Diggelen OP, et al. A fluorimetric enzyme assay for the diagnosis of Morquio disease type A (MPS IV A). Clin Chim Acta. 1990;187(2):131-139. 